\title{
Whole genome wide expression profiles of Vitis amurensis grape responding to downy mildew by using Solexa sequencing technology
}

\author{
Jiao $\mathrm{Wu}^{1,2+}$, Yali Zhang ${ }^{1 \dagger}$, Huiqin Zhang ${ }^{1}$, Hong Huang ${ }^{3}$, Kevin M Folta ${ }^{2}$, Jiang Lu $^{1,4^{*}}$
}

\begin{abstract}
Background: Downy mildew (DM), caused by pathogen Plasmopara viticola (PV) is the single most damaging disease of grapes (Vitis L.) worldwide. However, the mechanisms of the disease development in grapes are poorly understood. A method for estimating gene expression levels using Solexa sequencing of Type I restrictionendonuclease-generated cDNA fragments was used for deep sequencing the transcriptomes resulting from PV infected leaves of Vitis amurensis Rupr. cv. Zuoshan-1. Our goal is to identify genes that are involved in resistance to grape DM disease.

Results: Approximately 8.5 million (M) 21-nt CDNA tags were sequenced in the CDNA library derived from PV pathogen-infected leaves, and about 7.5 M were sequenced from the cDNA library constructed from the control leaves. When annotated, a total of 15,249 putative genes were identified from the Solexa sequencing tags for the infection (INF) library and 14,549 for the control (CON) library. Comparative analysis between these two cDNA libraries showed about $0.9 \%$ of the unique tags increased by at least five-fold, and about $0.6 \%$ of the unique tags decreased more than five-fold in infected leaves, while $98.5 \%$ of the unique tags showed less than five-fold difference between the two samples. The expression levels of 12 differentially expressed genes were confirmed by Real-time RT-PCR and the trends observed agreed well with the Solexa expression profiles, although the degree of change was lower in amplitude. After pathway enrichment analysis, a set of significantly enriched pathways were identified for the differentially expressed genes (DEGs), which associated with ribosome structure, photosynthesis, amino acid and sugar metabolism.
\end{abstract}

Conclusions: This study presented a series of candidate genes and pathways that may contribute to DM resistance in grapes, and illustrated that the Solexa-based tag-sequencing approach was a powerful tool for gene expression comparison between control and treated samples.

\section{Background}

Downy mildew of grapes occurs in most parts of the world where grapes are grown, but favors those regions that experience warm, wet conditions during the vegetative growth of the vine. A major outbreak of the disease can cause severe losses in yield and berry quality. Symptoms of DM are usually first noticed on leaves as yellowish and later oily lesions on the leaf's upper surface with a 'downy' mass observed on the corresponding

\footnotetext{
* Correspondence: j.lu.cau@gmail.com

+ Contributed equally

'College of Food Science and Nutritional Engineering, China Agricultural University, Beijing, 100083, China

Full list of author information is available at the end of the article
}

underside of the leaf. It can also cause deformation of shoots, tendrils, inflorescences and clusters of young berries. Berries become less susceptible as they mature, however rachis infection can spread into the older fruit which leads to direct crop loss by shelling of berries [1].

Downy mildew is caused by the pathogen Plasmopara viticola (PV). Primary infection begins with the overwintering oospore on infected leaves or plant litter in the soil that germinates in the spring and produces a sporangium [2]. When plant parts are covered with a film of moisture from rain or irrigation, the sporangium releases small swimming spores (zoospores) that are then spread by splashing water. The spores can germinate by producing a germ tube that enters the green
C Biomed Central

() 2010 Wu et al; licensee BioMed Central Ltd. This is an Open Access article distributed under the terms of the Creative Commons Attribution License (http://creativecommons.org/licenses/by/2.0), which permits unrestricted use, distribution, and reproduction in any medium, provided the original work is properly cited. 
tissue (including leaves, inflorescences, bunches and young berries) through the stomates [3]. Secondary infection, which is the major source of disease spread, produces spores that may be mobilized by wind and rain to establish new infection sites. The cycle ends with the sexual production of over-wintering oospores [2].

Different genotypes of grapes show varying level of resistance to $\mathrm{PV}$, ranging from susceptible $V$. vinifera, to the moderately resistant $V$. rupestris and $V$. amurensis, $V$. cinerea, $V$. riparia and $V$. candicans, to the totally resistant Muscadinia rotundifolia [4-6]. The world-wide grape industry relies predominantly on $V$. vinifera, which requires chemical protection to produce healthy fruits. However, such chemicals may have negative environmental impacts and/or pose risk to human health. A promising alternative strategy that could simultaneously improve grape health and limit chemical use is to identify the unique genes or mechanisms from resistant species that could potentially confer resistance to the pathogen or lower presentation of symptoms. These elements may potentially be introduced into $V$. vinifera through long-term breeding efforts or transgenic methods. With this perspective, it is important to unravel the molecular basis of natural defense responses in resistant grapevines to DM challenge, including identification of the genetic processes that may contribute to resistance.

Responses to PV have been characterized in various resistant species. Mechanisms of resistance include induction of chemical barriers, initiation of processes that delay invasive growth of mycelia, or mechanisms that establish hypersensitive response after inoculation of PV [7-9]. Genetic and gene expression profiling studies have concluded that Rpv1, NPR1 homologs, and PR protein encoding genes contribute to the function of DM resistance in grapevines [10-12]. Others factors, including the amino acid beta-aminobutyric acid [13], and the proteins beta-1,3-Glucanase [14], stilbene synthase (STS) [15], phenylalanine ammonia lyase (PAL) [16], thaumatin-like proteins and chitinase [17] may also play an important role in DM resistance. Many attempts, including transgenic [18-21] and traditional breeding approaches $[10,22,23]$, have been undertaken to introgress resistance into $V$. vinifera genotypes.

To understand the mechanism(s) of the host resistance at the molecular level, a critical first step is to identify the transcripts that accumulate in response to the pathogen attack. In this study, "Zuoshan-1", a clonal selection from wild $V$. amurensis with cold hardiness and high resistance to DM [24], was employed to identify a set of candidate genes associated with DM resistance using Solexa sequencing technology. Solexa sequencing is a technology capable of obtaining novel information for wholegenome-wide transcript expression without prior sequence knowledge. This report presents the finding of these tests.

\section{Results}

Inoculation and symptom development

The fourth unfolded leaf from the shoot apex of "Zuoshan-1" was inoculated with PV. No visible symptoms were observed in the first 4 days (Figure 1a and $1 b)$. The 'downy' mass was obviously observed on the 6th day (Figure 1c) and exacerbated on the 8th day (Figure 1d). Oil spots emerged gradually on the site of pathogen and the spores did not spread to the other healthy tissues 18 days after inoculation (Figure 1e and 1f).

\section{Tag identification and quantification}

A total of 8,549,948 and 7,527,499 tags were sequenced in infected (INF) and control (CON) libraries, respectively (Table 1). After filtering out low quality tags (tags containing ' $\mathrm{N}$ ' and adaptor sequences), 8,474,583 and $7,525,307$ tags (noted herein as "clean" tags) remained in INF and CON libraries. To increase the robustness of the approach, single-copy tags in the two libraries (247,900 in INF and 253,156 in CON library) were excluded from further analysis. As a result, a total of $8,226,683$ and $7,272,151$ clean tags remained from the two libraries, from which 233,653 (INF) and 203,514 (CON) unique tags were obtained. There were 30,139 more unique tags in the INF than in the CON library, possibly representing genes related to pathogen interaction and symptom development. The percentage of unique tags rapidly declined as copy number increased, indicating only a small portion of the transcripts were expressed at high level in the conditions tested.

\section{Depth of sampling}

Saturation of the library is determined by identification of unique tags. Sequencing reaches saturation when no new unique tags are detected. The results shown in Figure 2 indicate that INF and CON libraries were sequenced to saturation, producing a full representation of the transcripts in the conditions tested. In both libraries fewer unique tags were identified as the number of sequencing tags increases, reaching a plateau shortly after $6 \mathrm{M}$ tags were sequenced. No new unique tags were identified as the total tag number approached 8.5 $\mathrm{M}$ in INF library and 7.5 $\mathrm{M}$ in CON library.

\section{Annotation analysis of the unique tag}

The unique tags were compared against the genome and gene sequences of $V$. vinifera $\mathrm{cv}$. Pinot Noir [25] using blastn. Tags with a complete match or one base pair mismatch were considered further. The results in Table 2 show that a substantial proportion of tags 


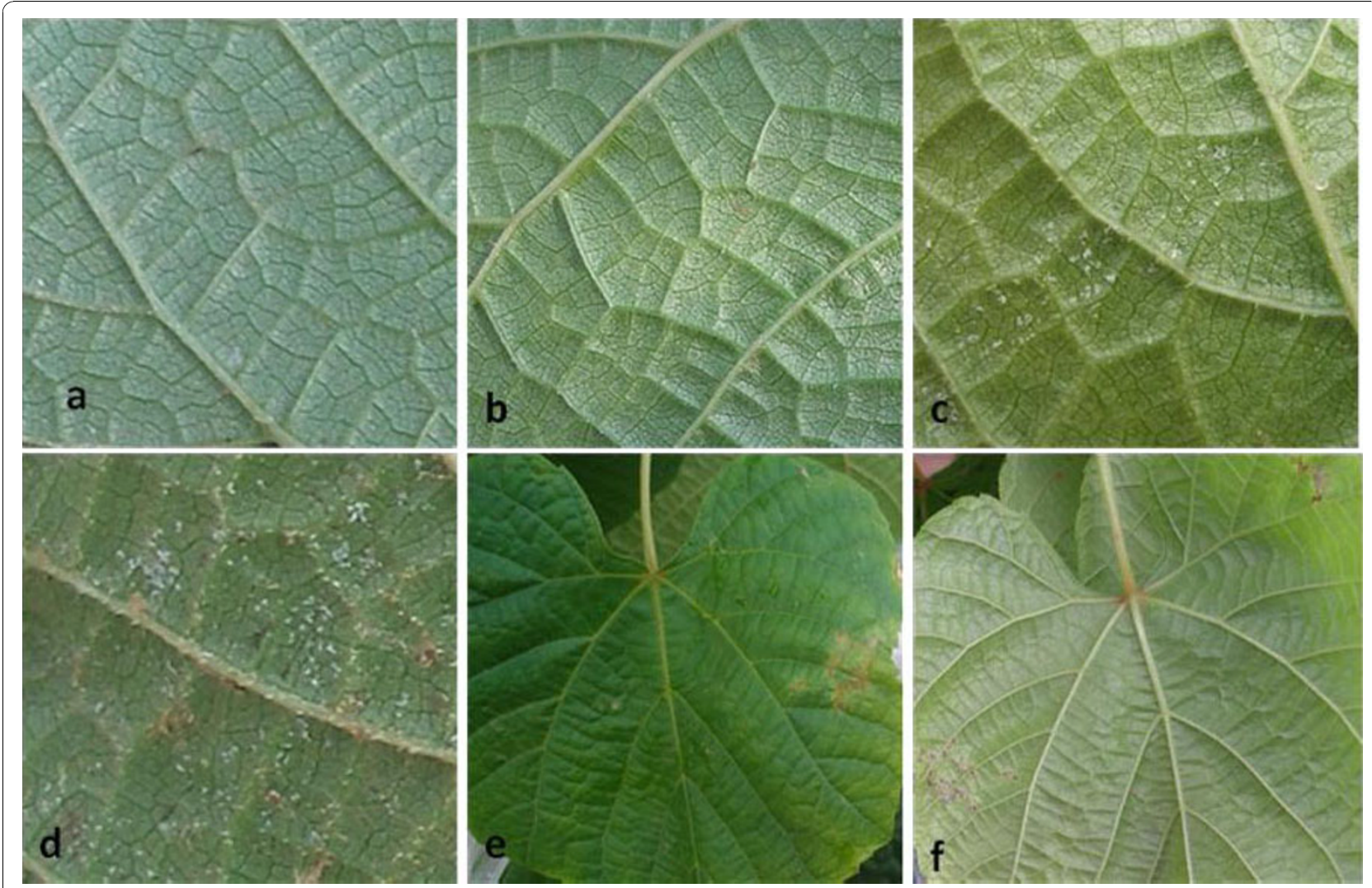

Figure 1 Symptom development on leaf surface of "Zuoshan-1" after PV infection. The fourth unfolded leaf from the shoot apex of "Zuoshan-1" was inoculated on (a) day 0 . Subsequent images depict the state of infection and symptom development on (b) day 4, (c) day 6, (d) day 8 and (e and f) $18 \mathrm{~d}$. Panel e shows the upper leaf and panel $f$ shows the lower leaf surface.

(81.60\% in INF library and $83.72 \%$ in CON library) matched to the "Pinot Noir" genome, and 91,638 (39.21\% of unique tags) and 83,079 (40.82\% of unique tags) in INF and CON library matched to 18,841 (61.91\%) and 18,068 (59.37\%) "Pinot Noir" genes. Further analysis revealed that 82,886 unique tags (35.47\%) in INF library and 75,290 (36.99\%) in CON library matched to only one gene sequence in the "Pinot Noir' genome (Table 2). These data indicated that

\section{Table 1 Solexa tags in the infected (INF) and control} (CON) libraries

\begin{tabular}{lrr}
\hline & INF & CON \\
\hline total tag & 8549948 & 7527499 \\
clean tag & 8474583 & 7525307 \\
clean tag copy number $=1$ & 247900 & 253156 \\
unique tag & 233653 & 203514 \\
unique tag copy number $>5$ & 98318 & 80345 \\
unique tag copy number $>10$ & 63202 & 51438 \\
unique tag copy number $>20$ & 39772 & 31441 \\
unique tag copy number $>50$ & 19776 & 14804 \\
unique tag copy number $>100$ & 10615 & 7701 \\
\hline
\end{tabular}

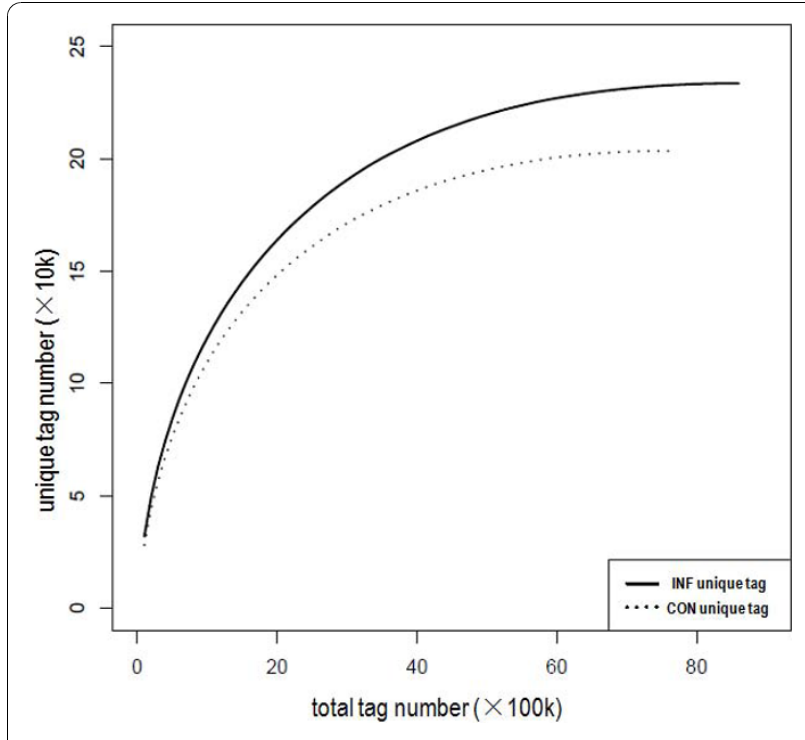

Figure 2 Accumulation of Solexa total tag and unique tag in the two libraries. New unique tag ("y" axis) of INF (solid line) and CON (broken line) libraries decreased as the solexa sequencing increased (" $x "$ axis). The total unique tag was 233,653 in INF and 203,514 in CON library. 
Table 2 Annotation of "Zuoshan-1" Solexa tags against the "Pinot Noir" genomic sequence

\begin{tabular}{lcccc}
\hline & \multicolumn{2}{c}{ INF } & \multicolumn{2}{c}{ CON } \\
\cline { 2 - 5 } & match to genome & match to gene & match to genome & match to gene \\
\hline unique tag & $190665(81.60 \%)^{*}$ & $91638(39.21 \%)^{*}$ & $170380(83.72 \%)^{*}$ & $83079(40.82 \%)^{*}$ \\
matched genes & & $18841(61.91 \%)^{\#}$ & $18068(59.37 \%)^{\#}$ \\
\hline unique tag matched to one gene & & $82886(35.47 \%)^{*}$ & $75290(36.99 \%)^{*}$ \\
matched genes & $15249(50.51 \%)^{\#}$ & $14549(47.81 \%)^{\#}$ \\
\hline
\end{tabular}

Note: *percentage of matched tags/total tags; ${ }^{*}$ percentage of matched genes/total assembled CDs of "Pinot Noir".

approximately $50 \%$ of transcripts predicted in grape are expressed in the infected or control leaves, with more transcripts present in the infected sample.

Tags with no homology to grape were compared with blastn to the VBI Microbial Database [26] containing genomic sequence information from Phytophthora sojae, Phytophthora infestans and Hyaloperonospora parasitica. There were 251 tags identified in INF library found to be identical to those of the oomycete during PV infection (additional file 1).

\section{Comparison of gene expression level between the two libraries}

Differences of tag frequencies that appeared in the INF and $\mathrm{CON}$ libraries were used for estimating gene expression levels in response to PV infection. The transcripts detected with at least two-fold differences in the two libraries are shown in Figure 3 (FDR $<0.001)$. The red dots $(3,125)$ and green dots $(1,847)$ represent transcripts higher or lower in abundance for more than two fold in INF library, respectively. The blue dots represent transcripts that differed less than two fold between the two libraries, which were arbitrarily designated as "no difference in expression". The DEGs with five fold or greater differences in accumulation were shown in Figure 4. A total of 513 genes (about $0.9 \%$ total unique tags) increased by at least five fold, and 167 genes (about $0.6 \%$ total unique tags) were decreased by at least five fold in the INF library, while the expression level of $98.5 \%$ unique tags was within five-fold difference between the two samples.

Of DEGs with differences greater than twenty fold (Table 3), 69 genes were present at higher levels in the INF library, 67 of which were associated with defense (6), transport (3), transcription (11), signal transduction (14) and metabolism (33). The highest DEG was phosphate-induced protein gene which was present at 229 fold of control levels. Among these highly expressed genes, many were associated with senescence, abiotic and biotic stresses.

Fifteen DEGs were less abundant in the INF library. Those present twenty fold or more in the CON library were also listed in Table 3, in which 13 genes were classified as defense (2) and metabolism (11), including genes encoding cytochrome P450 and PR proteins. The greatest differences between INF and CON DEGs were (-)-germacrene D synthase and immunoglobulin/major histocompatibility complex that both were present 164fold lower in the INF library than in the CON library.

\section{Real-time RT-PCR analysis}

In order to validate Solexa expression profiles, the steady-state transcript levels of 12 "defense related" genes were analyzed. Among them, seven genes (CHI4D, TL3, PR10, TIP2;1, CYSP, ERF4, STS5) were upregulated and five genes (THX, SHM1, HypP, GLO, $\mathrm{ClpP}$ ) were downregulated (Figure 5). Actin, tested to be stable in our previous work, was chosen as a reference gene for data normalization. The trend of RT-PCR based expression profiles among these selected genes was similar to those detected by Solexa-sequencing

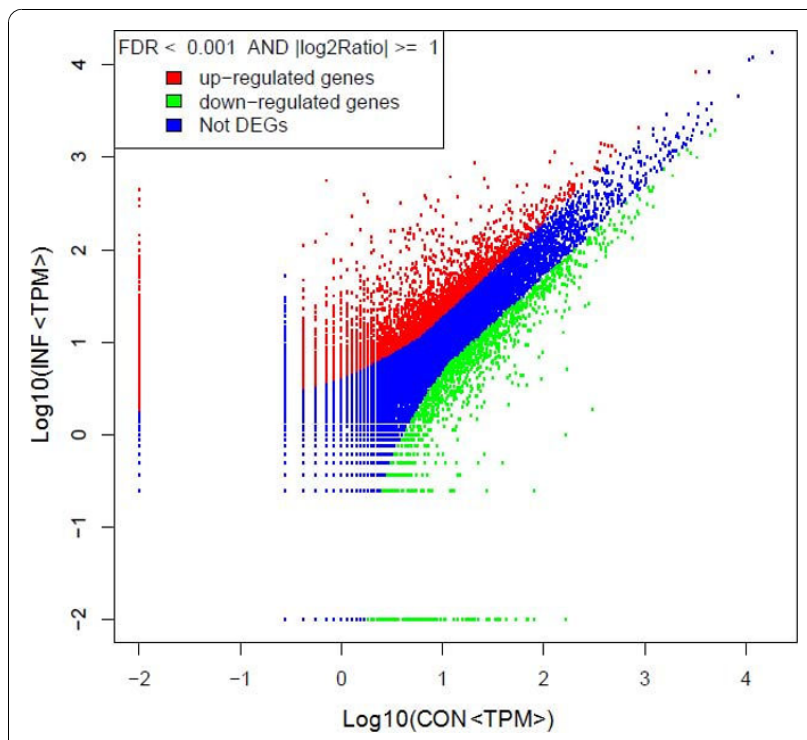

Figure 3 Comparision of gene expression level between the two libraries. For comparing gene expression level between the two libraries, each library was normalized to 1 million tags. Red dots represent transcripts more prevalent in the infected leaf library, green dots show those present at a lower frequency in the infected tissue and blue dots indicate transcripts that did not change significantly. The parameters "FDR $<0.001$ " and "log2 Ratio $\geq 1$ " were used as the threshold to judge the significance of gene expression difference. 


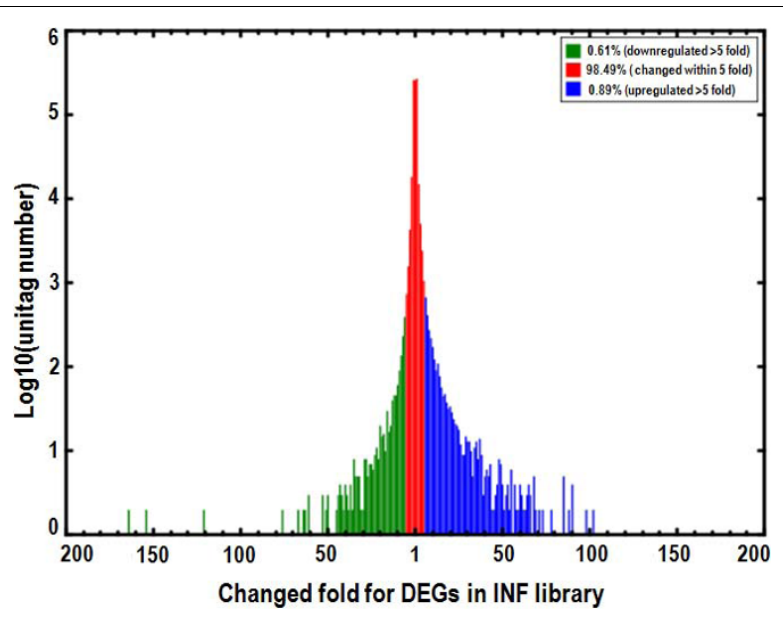

Figure 4 Differentially expressed tags in infected (INF) tissue library. The " $x$ " axis represents fold-change of differentially expressed unique tags in the INF library. The "y" axis represents the number of unique tags $(\log 10)$. Differentially accumulating unique tags with a 5-fold difference between libraries are shown in the red region $(98.49 \%)$. The blue $(0.89 \%)$ and green $(0.61 \%)$ regions represent unique tags that are up- and downregulated for more than 5 fold in the INF library, respectively.

based method. However, the scales of difference between the INF and CON were generally smaller in Real-time PCR (1-18 fold differences) than in those detected by the Solexa-sequencing based method (2 - 57 folds) (Table 4).

\section{Pathway enrichment analysis of DEGs}

The PV affected biological pathways were evaluated by enrichment analysis of DEGs. Significantly enriched metabolic pathways and signal transduction pathways were identified. A total of 115 pathways were affected by up- and 107 were affected by down-regulated DEGs, respectively (additional file 2 and 3). DEGs with pathway annotation were listed according to enrichment priority (additional file 4 and 5). The first ten enriched pathways were reported in Table 5. Pathways with $\mathrm{Q}$ value $<0.05$ are significantly enriched.

Ribosomal-associated proteins constituted the only significantly affected pathway for the upregulated DEGs $(\mathrm{Q}$ $<0.05)$. Other non-significant enriched pathways with large number of upregulated DEGs included amino sugar and nucleotide sugar metabolism, starch and sucrose metabolism, secondary metabolism, plant hormone biosynthesis, and splicesome associated proteins. There were more significantly enriched pathways (10) for the downregulated DEGs, which were involved in photosynthesis, as well as metabolism of folate, nicotinate, nicotinamide, fructose, mannose, pyruvate, polyketide sugar unit, and purines, along with alkaloids from histidine and purines.

\section{Discussion}

In this report Solexa sequencing technology, a highthroughput DNA sequencing approach, was utilized to estimate gene expression in libraries prepared from infected and control tissues. The results (Figure 2) provided estimates of gene expression as determined by the frequency that any given tag (representing a transcript) is sequenced. The data indicate that there is sufficient coverage depth to reach saturation, that is, a complete assessment of all transcripts present in the libraries. Theoretically, the rate of novel tag discovery should equal zero if all unique tags of the initial sample had been sequenced. However, this number might be slightly higher because new tags may be added due to the accumulation of sequencing errors as the size of the library increased [27]. Strict filtering and conservative matching allows recognition of erroneous tags, which are then disregarded. All of these precepts may contribute to a loss of substantial sequence information. However, loss of some data potentially made the results more conservative, revealing only robust and bona fide differences. Moreover, the total number of tags after stringent filtering was sufficient for annotation to the reference genes in the grape genome sequence. Theoretically, tags should be generated by NlaIII from the 3'-most ends of transcripts, but almost $50 \%$ of tags from other NlaIII sites were also generated in our result. Since only one tag could be generated in each transcript from any NlaIII site in a cDNA, these other NlaIII tags represented a given gene redundantly in the expression profile. This phenomenon accounts for the inflated number of unique tags generated (about 200,000) relative to that of the annotated grape genome (about 30,000). These other tags may also arise because of alternative splicing or incomplete enzyme digestion.

The results represent the first large-scale investigation of the gene expression in DM analysis of grapevine. Polesani et al [28] reported 804 transcripts identified in PV infected leaves of susceptible cultivar "Riesling" using cDNA-AFLP. Figueiredo et al [29] found 121 transcripts, representing 29 unique gene differentially expressed between two V. vinifera cultivars "Regent" and "Trincadeira" (resistant and susceptible to fungi, respectively) by cDNA microarray. In the current study, 15,249 putative genes were identified among the Solexa sequencing tags for the INF library and 14,549 for the CON library.

The steady-state transcript level for a set of selected genes was confirmed by Real-time RT-PCR. Although the differences in gene expression did not match the magnitude of those detected by Solexa-based sequencing method, the trends of up- and down- regulation were similar. The lower expression level detected by Real-time 
Table 3 List of DEGs changed for 20 fold and more in INF library

\begin{tabular}{|c|c|c|c|c|c|}
\hline Gene & Annotation & Stress related function & Accession & Identity & Fold \\
\hline \multicolumn{6}{|c|}{ Upregulated genes } \\
\hline \multicolumn{6}{|l|}{ Defence } \\
\hline GSVIVT00025506001 & $\begin{array}{l}\text { polygalacturonase-inhibiting protein [Vitis labrusca } \\
\text { x Vitis Riparia] }\end{array}$ & $\begin{array}{l}\text { inhibits fungal } \\
\text { endopolygalacturonases }\end{array}$ & ACS16072.1 & $333 / 333(100 \%)$ & 60 \\
\hline GSVIVT00001105001 & thaumatin-like protein [Vitis vinifera] & $\begin{array}{l}\text { pathogen defence; drought } \\
\text { and heat combination }\end{array}$ & AAQ10092.1 & $217 / 225(96 \%)$ & 57 \\
\hline GSVIVT00017370001 & $\begin{array}{l}\text { harpin-induced protein-related/HIN1-related/ } \\
\text { harpin-responsive protein-related [Arabidopsis } \\
\text { thaliana] }\end{array}$ & pathogen defence; senescence & NP_565634.1 & $141 / 267(52 \%)$ & 33 \\
\hline GSVIVT00002965001 & TMV response-related protein [Zea mays] & $\begin{array}{l}\text { Tobacco Mosaic Virus } \\
\text { response }\end{array}$ & ACG48457.1 & $39 / 91(42 \%)$ & 32 \\
\hline GSVIVT00005362001 & glutaredoxin [Populus trichocarpa] & senescence & EEE75685.1 & $91 / 155(58 \%)$ & 29 \\
\hline GSVIVT00024683001 & beta-glucosidase [Rosa hybrid cultivar] & activation of phytoanticipins & BAG13451.1 & $382 / 531(71 \%)$ & 21 \\
\hline \multicolumn{6}{|l|}{ Transport } \\
\hline GSVIVT00001094001 & $\begin{array}{l}\text { multidrug resistance pump, putative [Ricinus } \\
\text { communis] }\end{array}$ & fungal resistance & EEF51093.1 & $407 / 509$ (79\%) & 121 \\
\hline GSVIVT00015121001 & $\begin{array}{l}\text { mitochondrial dicarboxylate carrier protein, } \\
\text { putative [Ricinus Communis] }\end{array}$ & aluminum tolerance & EEF48606.1 & $271 / 324(83 \%)$ & 38 \\
\hline GSVIVT00030447001 & $\begin{array}{l}\text { multidrug resistance protein } A B C \text { transporter family } \\
\text { protein [Populus Trichocarpa] }\end{array}$ & $\begin{array}{l}\text { Senescence; drought and heat } \\
\text { combination }\end{array}$ & EEE80779.1 & $64 / 194(32 \%)$ & 25 \\
\hline \multicolumn{6}{|l|}{ Signal transduction } \\
\hline GSVIVT00030628001 & $\begin{array}{l}\text { leucine-rich repeat receptor-like protein kinase } \\
\text { [Nicotiana tabacum] }\end{array}$ & senescence & AAF66615.1 & $644 / 923(69 \%)$ & 145 \\
\hline GSVIVT00006178001 & FERONIA receptor-like kinase [Arabidopsis thaliana] & defence, stresses & ABT18100.1 & $317 / 621(51 \%)$ & 56 \\
\hline GSVIVT00019504001 & MAP3K-like protein kinase [Arabidopsis thaliana] & $\begin{array}{l}\text { disease resistance, drought } \\
\text { and heat combination }\end{array}$ & CAB16796.1 & $184 / 359(51 \%)$ & 52 \\
\hline GSVIVT00002706001 & calmodulin-binding protein [Arabidopsis thaliana] & senescence & NP_565379.1 & $21 / 45(46 \%)$ & 39 \\
\hline GSVIVT00020989001 & $\begin{array}{l}\text { calcium-binding EF hand family protein } \\
\text { [Arabidopsis thaliana] }\end{array}$ & $\begin{array}{l}\text { defence related; senescence; } \\
\text { drought and heat } \\
\text { combination }\end{array}$ & NP_568568.1 & $81 / 166(48 \%)$ & 35 \\
\hline GSVIVT00029809001 & $\begin{array}{l}\text { ethylene-regulated transcript } 2 \text { (ERT2) [Arabidopsis } \\
\text { thaliana] }\end{array}$ & senescence & CAB45883.1 & $96 / 204(47 \%)$ & 34 \\
\hline GSVIVT00036549001 & calmodulin-binding protein [Arabidopsis thaliana] & senescence & NP_565379.1 & 149/366 (40\%) & 28 \\
\hline GSVIVT00002973001 & calmodulin binding protein-like [Elaeis guineensis] & senescence & ABP04242.1 & $89 / 135(65 \%)$ & 27 \\
\hline GSVIVT00025017001 & $\begin{array}{l}\text { BRASSINOSTEROID INSENSITIVE 1-associated } \\
\text { receptor kinase } 1 \text { precursor, putative [Ricinus } \\
\text { communis] }\end{array}$ & disease, cell death & EEF29110.1 & $415 / 639(64 \%)$ & 26 \\
\hline GSVIVT00000612001 & nodulin-like protein [Arabidopsis thaliana] & $\begin{array}{l}\text { drought and heat } \\
\text { combination }\end{array}$ & AAC28987.1 & $397 / 550(72 \%)$ & 23 \\
\hline GSVIVT00033036001 & $\begin{array}{l}\text { RING-H2 subgroup RHE protein [Populus tremula } x \\
\text { Populus alba] }\end{array}$ & $\begin{array}{l}\text { drought and heat } \\
\text { combination }\end{array}$ & AAW33880.1 & $168 / 296(56 \%)$ & 22 \\
\hline GSVIVT00009150001 & PAR-1a [Nicotiana tabacum] & potato virus Y, SAR induce & CAA58733.1 & 127/178 (71\%) & 22 \\
\hline GSVIVT00027614001 & $\begin{array}{l}\text { receptor-protein kinase-like protein [Arabidopsis } \\
\text { thaliana] }\end{array}$ & $\begin{array}{l}\text { drought and heat } \\
\text { combination }\end{array}$ & BAA98098.1 & $632 / 849(74 \%)$ & 20 \\
\hline GSVIVT00030574001 & $\begin{array}{l}\text { leucine-rich repeat receptor-like protein kinase } \\
\text { [Arabidopsis thaliana] }\end{array}$ & senescence & ACN59244.1 & $317 / 611(51 \%)$ & 20 \\
\hline \multicolumn{6}{|l|}{ Transcription } \\
\hline GSVIVT00014947001 & zinc-finger protein 1 [Datisca glomerata] & defence, stresses & AAD26942.1 & $144 / 246(58 \%)$ & 60 \\
\hline GSVIVT00016398001 & $\begin{array}{l}\text { dehydration-responsive element binding protein } 3 \\
\text { [Glycine max] }\end{array}$ & biotic and abiotic stresses & ABB36646.1 & $116 / 187(62 \%)$ & 52 \\
\hline GSVIVT00007409001 & DRE-binding protein 3b [Gossypium hirsutum] & $\begin{array}{l}\text { drought and heat } \\
\text { combination }\end{array}$ & ABB45861.1 & $134 / 237(56 \%)$ & 22 \\
\hline GSVIVT00020131001 & basic helix-loop-helix protein [Nicotiana tabacum] & senescence & BAF30984.1 & $105 / 228(46 \%)$ & 33 \\
\hline GSVIVT00001092001 & $\begin{array}{l}\text { Dehydration-responsive element-binding protein } \\
1 \mathrm{~F} \text {, putative [Ricinus communis] }\end{array}$ & $\begin{array}{l}\text { phytohormone, pathogen and } \\
\text { environmental stresses }\end{array}$ & EEF51090.1 & $143 / 242(59 \%)$ & 30 \\
\hline GSVIVT00007410001 & CBF4 transcription factor [Vitis vinifera] & cold stress & ABE96792.1 & $218 / 218(100 \%)$ & 30 \\
\hline GSVIVT00016403001 & jasmonate ZIM domain 1 [Catharanthus roseus] & wounding; herbivory; salinity & ACM89457.1 & $131 / 275(47 \%)$ & 27 \\
\hline
\end{tabular}




\section{Table $\mathbf{3}$ List of DEGs changed for $\mathbf{2 0}$ fold and more in INF library (Continued)}

\begin{tabular}{|c|c|c|c|c|}
\hline GSVIVT00028041001 & $\begin{array}{l}\text { AP2 domain class transcription factor [Malus } \mathrm{x} \\
\text { domestica] }\end{array}$ & $\begin{array}{l}\text { senescence; drought and heat } \\
\text { combination }\end{array}$ & ADE41117.1 & $172 / 327(52 \%)$ \\
\hline GSVIVT00027444001 & $\begin{array}{l}\text { GRAS family transcription factor [Populus } \\
\text { trichocarpa] }\end{array}$ & chitin response & EEE95719.1 & $446 / 586(76 \%)$ \\
\hline GSVIVT00006790001 & $\begin{array}{l}\text { basic helix-loop-helix (bHLH) family protein } \\
\text { [Arabidopsis thaliana] }\end{array}$ & $\begin{array}{l}\text { fugal resistance related; } \\
\text { senescence }\end{array}$ & NP_568850.1 & $152 / 239(63 \%)$ \\
\hline GSVIVT00002446001 & $\begin{array}{l}\text { WRKY transcription factor } 21 \text { [Populus tomentosa } \mathrm{x} \\
\text { P. bolleana] }\end{array}$ & senescence,stresses & ACV92023.1 & 196/364 (53\%) \\
\hline \multicolumn{5}{|l|}{ Metabolism } \\
\hline GSVIVT00015203001 & $\begin{array}{l}\text { putative phosphate-induced protein [Nicotiana } \\
\text { tabacum] }\end{array}$ & unidentified & BAA33810.1 & $243 / 317(76 \%)$ \\
\hline GSVIVT00016518001 & salt responsive protein 2 [Solanum lycopersicum] & $\begin{array}{l}\text { drought and heat } \\
\text { combination }\end{array}$ & ACG50004.1 & $309 / 464(66 \%)$ \\
\hline GSVIVT00024884001 & $\begin{array}{l}\text { S-adenosyl-L-methionine:salicylic acid carboxyl } \\
\text { methyltransferase [Chimonanthus praecox] }\end{array}$ & biotic and abotic stresses & ABU88887.2 & 191/377 (50\%) \\
\hline GSVIVT00024408001 & potein-binding protein, putative [Ricinus communis] & unidentified & EEF27653.1 & $393 / 605(64 \%)$ \\
\hline GSVIVT00028930001 & $\begin{array}{l}\text { ubiquitin-protein ligase, putative [Ricinus } \\
\text { communis] }\end{array}$ & senescence & EEF42248.1 & $357 / 602(59 \%)$ \\
\hline GSVIVT00014730001 & cytochrome P450 [Populus trichocarpa] & $\begin{array}{l}\text { senescence; drought and heat } \\
\text { combination }\end{array}$ & EEE73840.1 & $261 / 453(57 \%)$ \\
\hline GSVIVT00000988001 & 9-cis-epoxycarotenoid dioxygenase 1 [Vitis vinifera] & senescence; defence & AAR11193.1 & $602 / 610(98 \%)$ \\
\hline GSVIVT00023009001 & ATPP2-A2, putative [Ricinus communis] & unidentified & EEF38353.1 & 114/158 (72\%) \\
\hline GSVIVT00014704001 & $\begin{array}{l}\text { putative integral membrane protein [Cyanothece } \\
\text { sp. CCY0110] }\end{array}$ & unidentified & EAZ88012.1 & $53 / 176(30 \%)$ \\
\hline GSVIVT00018424001 & tropinone reductase, putative [Ricinus communis] & $\begin{array}{l}\text { senescence; drought and heat } \\
\text { combination }\end{array}$ & EEF38138.1 & 194/264 (73\%) \\
\hline GSVIVT00032938001 & $\begin{array}{l}\text { aspartic proteinase nepenthesin-1 precursor, } \\
\text { putative [Ricinus communis] }\end{array}$ & $\begin{array}{l}\text { phosphorus deficiency; salt } \\
\text { stress }\end{array}$ & EEF29846.1 & $306 / 441(69 \%)$ \\
\hline GSVIVT00024072001 & $\begin{array}{l}\text { protein phosphatase } 2 c \text {, putative }[\text { Ricinus } \\
\text { communis] }\end{array}$ & senescence & EEF41194.1 & 254/393 (64\%) \\
\hline GSVIVT00015200001 & $\begin{array}{l}\text { putative phosphate-induced protein [Capsicum } \\
\text { chinense] }\end{array}$ & unidentified & BAG16530.1 & $186 / 289(64 \%)$ \\
\hline GSVIVT00022245001 & f-box family protein [Populus trichocarpa] & senescence & EEE87327.1 & $139 / 345(40 \%)$ \\
\hline GSVIVT00016166001 & ATP-dependent DNA helicase [Brevibacillus brevis] & DNA repair & BAH41662.1 & $16 / 45(35 \%)$ \\
\hline GSVIVT00024387001 & $\begin{array}{l}\text { nucleic acid binding protein, putative [Ricinus } \\
\text { communis] }\end{array}$ & oxidative; ABA; abiotic stresses & EEF29282.1 & 102/164 (62\%) \\
\hline GSVIVT00024235001 & protein phosphatase $2 \mathrm{C}$ [Nicotiana tabacum] & senescence & CAC10358.1 & $257 / 429(59 \%)$ \\
\hline GSVIVT00035825001 & $\begin{array}{l}\text { ubiquitin-protein ligase, putative [Ricinus } \\
\text { communis] }\end{array}$ & senescence & EEF40124.1 & $572 / 719(79 \%)$ \\
\hline GSVIVT00019233001 & $\begin{array}{l}\text { TPA: isoflavone reductase-like protein } 3 \text { [Vitis } \\
\text { vinifera] }\end{array}$ & putative defence & CAI56332.1 & $301 / 319(94 \%)$ \\
\hline GSVIVT00014029001 & TPA_exp: cellulose synthase-like D1 [Oryza sativa] & unidentified & DAA01752.1 & $999 / 1171(85 \%)$ \\
\hline GSVIVT00007984001 & serine acetyltransferase [Nicotiana plumbaginifolia] & oxidative stress & AAR18403.1 & 179/307 (58\%) \\
\hline GSVIVT00036225001 & $\begin{array}{l}\text { Beta-expansin 1a precursor, putative [Ricinus } \\
\text { communis] }\end{array}$ & osmotic stress & EEF28288.1 & 207/259 (79\%) \\
\hline GSVIVT00017518001 & spotted leaf protein, putative [Ricinus communis] & $\begin{array}{l}\text { hypersensitive response; cell } \\
\text { death; senescence }\end{array}$ & EEF38265.1 & $243 / 402(60 \%)$ \\
\hline GSVIVT00007452001 & $\begin{array}{l}\text { wound-induced protein WIN2 precursor, putative } \\
\text { [Ricinus communis] }\end{array}$ & antifungal & EEF31100.1 & 142/197 (72\%) \\
\hline GSVIVT00002450001 & UDP-glucose:glucosyltransferase [Lycium barbarum] & $\begin{array}{l}\text { drought and heat } \\
\text { combination }\end{array}$ & BAG80556.1 & 293/464 (63\%) \\
\hline GSVIVT00036349001 & $\begin{array}{l}\text { glucose-1-phosphate adenylyltransferase, putative } \\
\text { [Ricinus communis] }\end{array}$ & $\begin{array}{l}\text { drought and heat } \\
\text { combination }\end{array}$ & EEF49428.1 & $412 / 531(77 \%)$ \\
\hline GSVIVT00028839001 & spotted leaf protein, putative [Ricinus communis] & $\begin{array}{l}\text { hypersensitive response; cell } \\
\text { death; senescence }\end{array}$ & EEF52025.1 & $385 / 674(57 \%)$ \\
\hline GSVIVT00009741001 & f-box family protein [Populus trichocarpa] & senescence & EEE86166.1 & 93/182 (51\%) \\
\hline GSVIVT00019669001 & galactinol synthase [Solanum lycopersicum] & $\begin{array}{l}\text { oxidative stress; drought; } \\
\text { salinity; chilling; heat shock }\end{array}$ & BAH98060.1 & 231/316 (73\%) \\
\hline
\end{tabular}


Table $\mathbf{3}$ List of DEGs changed for $\mathbf{2 0}$ fold and more in INF library (Continued)

\begin{tabular}{|c|c|c|c|c|c|}
\hline GSVIVT00030537001 & $\begin{array}{l}\text { senescence-associated protein, putative [Medicago } \\
\text { truncatula] }\end{array}$ & $\begin{array}{l}\text { Senescence; drought and heat } \\
\text { combination }\end{array}$ & ABD32641.1 & $99 / 144(68 \%)$ & 2 \\
\hline GSVIVT00001432001 & $\begin{array}{l}\text { protein phosphatase } 2 \mathrm{c} \text {, putative [Ricinus } \\
\text { communis] }\end{array}$ & $\begin{array}{l}\text { senescence; drought and heat } \\
\text { combination }\end{array}$ & EEF34881.1 & $319 / 389(82 \%)$ & \\
\hline GSVIVT00033193001 & galactinol synthase [Capsicum annuum] & $\begin{array}{l}\text { oxidative stress; drought; } \\
\text { salinity; chilling; heat shock }\end{array}$ & $\mathrm{ABQ} 44212.1$ & $239 / 315(75 \%)$ & \\
\hline GSVIVT00023109001 & $\begin{array}{l}\text { ATEXO7OH4 (exocyst subunit EXO70 family protein } \\
\text { H4); protein binding [Arabidopsis thaliana] }\end{array}$ & unidentified & NP_187563.1 & $331 / 585(56 \%)$ & \\
\hline \multicolumn{6}{|l|}{ various functions } \\
\hline GSVIVT00017533001 & PREDICTED: hypothetical protein [Vitis vinifera] & unidentified & XP_002279648.1 & $500 / 500(100 \%)$ & \\
\hline GSVIVT00020834001 & CW14 [Arabidopsis thaliana] & unidentified & BAA87958.1 & $300 / 533(56 \%)$ & \\
\hline \multicolumn{6}{|c|}{ Downregulated genes } \\
\hline \multicolumn{6}{|l|}{ Defence } \\
\hline GSVIVT00016961001 & $\begin{array}{l}\text { Immunoglobulin/major histocompatibility complex } \\
\text { [Medicago truncatula] }\end{array}$ & disease resistance & ABP03850.1 & $426 / 672(63 \%)$ & 1 \\
\hline GSVIVT00014282001 & $\begin{array}{l}\text { pathogenesis-related like protein [Arabidopsis } \\
\text { thaliana] }\end{array}$ & defence & AAM66077.1 & $117 / 215(54 \%)$ & -6 \\
\hline \multicolumn{6}{|l|}{ Metabolism } \\
\hline GSVIVT00027449001 & (-)-germacrene D synthase [Vitis vinifera] & wounding; methyl jasmonate & AAS66357.1 & $500 / 553(90 \%)$ & -164 \\
\hline GSVIVT00027451001 & (-)-germacrene D synthase [Vitis vinifera] & wounding; methyl jasmonate & AAS66357.1 & $503 / 557(90 \%)$ & -150 \\
\hline GSVIVT00027450001 & (-)-germacrene D synthase [Vitis vinifera] & wounding; methyl jasmonate & AAS66357.1 & $274 / 319(85 \%)$ & -5 \\
\hline GSVIVT00027456001 & (-)-germacrene D synthase [Vitis vinifera] & wounding; methyl jasmonate & AAS66357.1 & $454 / 545(83 \%)$ & -22 \\
\hline GSVIVT00014725001 & cytochrome P450 [Populus trichocarpa] & pathogen induced & EEE73840.1 & 299/511 (58\%) & -4 \\
\hline GSVIVT00014727001 & cytochrome P450 [Populus trichocarpa] & pathogen induced & EEE73840.1 & $269 / 447(60 \%)$ & -3 \\
\hline GSVIVT00007099001 & thioredoxin $\times$ [Populus trichocarpa] & $\begin{array}{l}\text { defence; abiotic stresses, } \\
\text { senescence }\end{array}$ & EEE90516.1 & $98 / 117(83 \%)$ & \\
\hline GSVIVT00008711001 & beta-cyanoalanine synthase [Betula pendula] & cyanide metabolism & AAN86822.1 & $311 / 352(88 \%)$ & 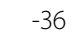 \\
\hline GSVIVT00037489001 & non-specific lipid transfer protein [Vitis vinifera] & defence related & ABA29446.1 & 119/119 (100\%) & -2 \\
\hline GSVIVT00029445001 & expansin [Vitis labrusca $\times$ Vitis vinifera] & defence related & BAC66695.1 & $252 / 252(100 \%)$ & -2 \\
\hline GSVIVT00006300001 & $\begin{array}{l}\text { UDP-glucosyltransferase, putative [Ricinus } \\
\text { communis] }\end{array}$ & defence related & EEF47681.1 & $268 / 466(57 \%)$ & \\
\hline \multicolumn{6}{|l|}{ various functions } \\
\hline GSVIVT00005678001 & male sterility-related protein [Linum usitatissimum] & unidentified & ACA28679.1 & $260 / 503(51 \%)$ & \\
\hline GSVIVT00032599001 & hypothetical protein [Vitis vinifera] & unidentified & XP_002284962.1 & $368 / 368(100 \%)$ & \\
\hline
\end{tabular}

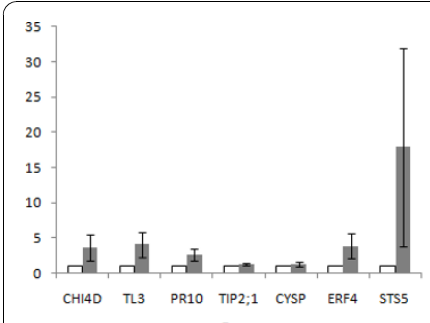

a

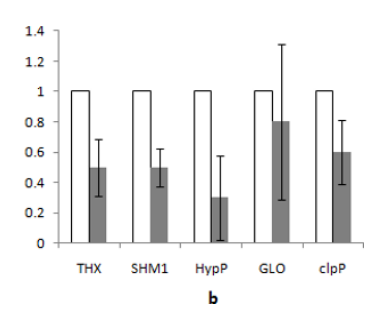

Figure 5 Real-time RT-PCR analysis for twelve differentially expressed genes. Real-time RT-PCR analysis for twelve transcripts in control (white) and infected (gray) samples, including (a) seven more abundant in the INF library and (b) five less prevalent in the INF library as identified by Solexa expression profile. All data were normalized to the actin expression level. Data represent fold change of $\mathrm{RQ}$ (relative quantification) in infected vs. control samples. Bars represent $\mathrm{RQ}$ standard deviation calculated from three biological replicates.
RT-PCR could be due to the difference of sensitivity between the two technologies. Solexa sequencing has been documented to be more sensitive for estimation of gene expression, especially for low-abundance transcripts compared to microarrays and Real-time RT-PCR [30]. The difference could also be attributed to different inoculation seasons and developmental stages of the grapevines. The materials used for the Solexa sequencing method were obtained from materials inoculated and harvested in September, while materials used for the Real-time RT- PCR analyses were obtained from plants inoculated and harvested in June.

Due to the sensitivity of Solexa sequencing technology, many rare transcripts were detected. Among 536 transcripts present predominantly $(<2-20$ fold $)$ in the INF library, 89 were not detected in the CON library at all. These genes were predicted to be involved in many plant 
Table 4 Genes selected for Real-time RT-PCR

\begin{tabular}{|c|c|c|c|c|c|c|}
\hline Gene & Description & Forward primer & Reverse primer & $\begin{array}{l}\text { Target } \\
\text { size }\end{array}$ & $\begin{array}{r}\text { Solexa } \\
\text { fold }\end{array}$ & $\begin{array}{l}\text { RT- } \\
\text { PCR } \\
\text { fold }\end{array}$ \\
\hline $\mathrm{CHI} / 4 \mathrm{D}$ & V. vinifera class IV chitinase (gb|AF532966.1) & TCCCACGTTCCCCCTTCT & GTAGCTTGGCTGCCATTTTTG & 59 & 11 & \\
\hline TL3 & $\begin{array}{l}\text { V.vinifera thaumatin-like protein (gb| } \\
\text { AF532965.1) }\end{array}$ & ACCCCACTCCAACCATCAAG & GATTTTGCAGAGGCCCATTG & 59 & 57 & \\
\hline PR10 & $\begin{array}{l}\text { Tamnara Tam-RP10 pathogenesis-related } \\
\text { protein } 10 \text { (dbj|AB372561.1) }\end{array}$ & GGTCAGGCCTCAAGCTATCAA & CAGGGCCTCCGTCTCCTT & 56 & 10 & \\
\hline TIP2;1 & V. vinifera aquaporin TIP2;1 (gb|EF364439.1) & GCATCATTGCACCCATTGC & GCCTGCAGCCAGGATGTT & 59 & 6 & \\
\hline CYSP & V. vinifera cysteine protease (gb|EU280160.1) & CCTCGCAGGAGGAGCACGAT & CCGGCGCAGGTTTGC & 54 & 2 & \\
\hline ERF4 & $\begin{array}{l}\text { V. aestivalis putative ethylene response factor } \\
4 \text { (gb|AY484580.1) }\end{array}$ & TCATCACTGCAACTCATCCA & TTACAATCTTCGGCCTCTGA & 101 & 11 & \\
\hline STS5 & V. vinifera stilbene synthase5 (gb|AY670312.1) & CGCTCAAGGGAGGAAAGACA & AGCCAAACAAAACACCCCAATC & 58 & 12 & 18 \\
\hline $\mathrm{THX}$ & $\begin{array}{l}\text { thioredoxin } \times \text { [Populus trichocarpa] } \\
(\text { XP_002310066.1) }\end{array}$ & TGCTCAGGAATACGGGGACAGA & TCGCGGGTTTGCATCAT & 61 & -39 & , \\
\hline SHM1 & $\begin{array}{l}\text { A. thaliana serine hydroxymethyl transferase } \\
1 \text { (ref|NM_119954.3) }\end{array}$ & TGTTCATCAGGTCAGCCAGTTT & TGCGTCGAATTGCAGCAAGAT & 63 & -2 & - \\
\hline HypP & Hypothetical protein LOC100264849 & TGCCCCTACCCTTGTGACA & GATCAAAATGGCTCATCGGAA & 58 & -5 & \\
\hline GLO & $\begin{array}{l}\text { V. pseudoreticulata glyoxal oxidase (gb| } \\
\text { D201181.1) }\end{array}$ & TCCCAACGCCGGTATAGC & ACCGTGCCGTAACGTGTGA & 54 & -5 & \\
\hline ClpP & $\begin{array}{l}\text { Carica papaya ATP-dependent Clp protease } \\
\text { proteolytic subunit (gb|DQ159405.1|) }\end{array}$ & GGGCGCCGGACAAGA & TTTGCAAATCATCCCTAATGGA & 55 & -2 & \\
\hline
\end{tabular}

biological processes, including defense. For example, genes encoding cinnamyl alcohol dehydrogenase, lipaselike protein, glutathione synthetase, GDSL-motif lipase, ankyrin repeat family protein, serine hydrolase, prolinerich cell wall protein and multicopper oxidase were previously described as plant defense-related genes. Other rare transcripts detected by Solexa technology were predicted to function in signal transduction (protein kinase, calcium ion binding protein, wall-associated kinase), transport (type IIIa membrane protein, ATP

Table 5 List of first ten pathways for up- and downregulated EDGs

\begin{tabular}{|c|c|c|c|c|}
\hline Pathway term & Pathway ID & DEGs tested & $P$ value & $Q$ value \\
\hline \multicolumn{5}{|l|}{ Pathways for upregulated DEGs } \\
\hline Ribosome & ko03010 & $53(4.36 \%)$ & 0.0004 & 0.0406 \\
\hline Amino sugar and nucleotide sugar metabolism & ko00520 & $25(2.06 \%)$ & 0.0010 & 0.0563 \\
\hline Glycolysis/Gluconeogenesis & ko00010 & $28(2.3 \%)$ & 0.0043 & 0.1660 \\
\hline Biosynthesis of alkaloids derived from histidine and purine & ko01065 & $31(2.55 \%)$ & 0.0126 & 0.3636 \\
\hline Biosynthesis of alkaloids derived from ornithine, lysine and nicotinic acid & ko01064 & $35(2.88 \%)$ & 0.0207 & 0.4459 \\
\hline Starch and sucrose metabolism & ko00500 & $49(4.03 \%)$ & 0.0233 & 0.4459 \\
\hline Biosynthesis of alkaloids derived from shikimate pathway & ko01063 & $39(3.21 \%)$ & 0.0361 & 0.5868 \\
\hline N-Glycan biosynthesis & ko00510 & $10(0.82 \%)$ & 0.0528 & 0.5868 \\
\hline Fructose and mannose metabolism & ko00051 & $14(1.15 \%)$ & 0.0560 & 0.5868 \\
\hline Selenoamino acid metabolism & ko00450 & $11(0.91 \%)$ & 0.0587 & 0.5868 \\
\hline \multicolumn{5}{|l|}{ Pathways for downregulated DEGs } \\
\hline Photosynthesis & ko00195 & $20(3.14 \%)$ & $9.9613 e-06$ & 0.0011 \\
\hline Photosynthesis - antenna proteins & ko00196 & $6(0.94 \%)$ & $4.2252 \mathrm{e}-05$ & 0.0023 \\
\hline Folate biosynthesis & ko00790 & $5(0.78 \%)$ & 0.0002 & 0.0064 \\
\hline Nicotinate and nicotinamide metabolism & ko00760 & $5(0.78 \%)$ & 0.0007 & 0.0125 \\
\hline Fructose and mannose metabolism & ko00051 & $13(2.04 \%)$ & 0.0007 & 0.0125 \\
\hline Carbon fixation in photosynthetic organisms & ko00710 & $13(2.04 \%)$ & 0.0007 & 0.0125 \\
\hline Pyruvate metabolism & ko00620 & $14(2.2 \%)$ & 0.0014 & 0.0210 \\
\hline Polyketide sugar unit biosynthesis & ko00523 & $4(0.63 \%)$ & 0.0016 & 0.0210 \\
\hline Purine metabolism & ko00230 & $21(3.3 \%)$ & 0.0018 & 0.0215 \\
\hline Biosynthesis of alkaloids derived from histidine and purine & ko01065 & $21(3.3 \%)$ & 0.0025 & 0.0270 \\
\hline
\end{tabular}


binding protein, $\mathrm{D}$-galactonate transporter, peptide transporter), transcription (ccaat-binding transcription factor, AP2/ERF domain-containing transcription factor, mutator-like transposase-like protein), and protein metabolism (ubiquitin-protein ligase, $50 \mathrm{~S}$ ribosomal protein, Slocus-specific glycoprotein S13 precursor, Rab5-interacting protein). Two novel genes (nectar protein 1, vernalization-insensitive protein) and some genes encoding hypothetical proteins (LOC100244011, LOC100258240, LOC100249110) were also identified from the PVinduced rare DEGs. Among the 608 rare transcripts present more in CON than INF, 69 were not detected at all in the INF library. Most of these transcripts have predicated biological functions in growth regulation (growth regulator protein, A-type cyclin, auxin response factor 8 ), transport (ATP-binding cassette transporter, AWPM-19like membrane family protein, copper-transporting atpase p-type), signal transduction (serine-threonine protein kinase, leucine-rich repeat family protein, calciumbinding EF hand family protein, calcium-dependent phospholipid binding ), and metabolism (galacturonosyltransferase 6, methylenetetrahydrofolate dehydrogenase, iron ion binding/oxidoreductase, trehalose-6-phosphate synthase, senescence-associated protein).

Pathway enrichment analysis revealed the most significantly affected pathways during the PV infection in "Zuoshan-1". It is not surprising that the "ribosomerelated" pathway was the most affected for the DEGs more common in INF library. This finding implies that the grapevine utilizes new ribosomes or changes in ribosome components to help synthesize additional proteins, such as PR proteins, to protect itself from the pathogen attack. The second affected pathway was the "amino sugar and nucleotide sugar metabolism" pathway. In this pathway genes encoding chitinase were more prevalent in the INF than the CON library. In addition, genes required for cell wall biosynthesis were also affected, such as D-xylan synthase, UDP-glucose dehydrogenase, and UDP-glucose 4,6-dehydratase. These enzymes are involved in the interconversion of nucleotide sugars, and may regulate glycosylation patterns in response to pathogen, thereby linking signaling with primary metabolism and the dynamics of the extracellular matrix. The other noticeable pathways with a large amount of DEGs associated with PV infection were starch and sucrose metabolism, secondary metabolism, plant hormone biosynthesis, and splicesome-associated proteins. For DEGs less prevalent in infected vs. control libraries, there was significant enrichment for transcripts associated with photosynthesis. This result was similar to the reports of Polesani et al $[28,31]$. Photosystem I proteins (PsaA, PsaB, PsaC), photosystem II proteins (PsbB, PsbD, PsbO, PsbP, PsbS), cytochorme b6/f complex
(PetD, PetN) and F-type ATPase (beta, alpha, delta, a, b) were all substantially lower in abundance in INF libraries compared to CON libraries. The reduction of photosynthesis was possibly due to the increase of invertase activity in nucleotide sugar metabolism pathway. Invertase would cleave sucrose into hexose sugars and their accumulation inhibits the Calvin cycle.

It was observed that 251 tags identified in INF library were homologous to the oomycete, indicating that they may belong to PV transcripts, predictably noting the presence of the pathogen. Many of these putative PV transcripts corresponded to genes involved in protein metabolism (16S, 18S, 26S, $28 \mathrm{~S}$ and $60 \mathrm{~S}$ ribosomal protein subunits) as a requirement for protein synthesis in the pathogen during the plant-pathogen interaction. Many housekeeping genes (alpha-tubulin, elongation factor 1 alpha, ubiquitin and heat shock protein 70) and genes related to immune response (spike 1 protein and cyclophilin) were also detected. Several PV transcripts showed similarity to enzymes involved in carbohydrate and amino acid metabolism (chlorophyll apoprotein, aspartate aminotransferase, glutamine synthetase and hyaluronoglucosaminidase-4), energy production (ATP synthase subunit B, glyceraldehyde-3-phosphate dehydrogenase, phosphoenolpyruvate carboxykinase and nitrate reductase), and cellular transport (transportin 1, $\mathrm{K}^{+}$channel protein and calmodulin).

\section{Transcripts more abundant in infected leaves}

A set of transcripts were clearly more abundant in tissue arising after PV infection compared to control. This group possibly contains elements that confer resistance to the spread of the pathogen in "Zuoshan-1". Among these transcripts, those expressed at a relatively high level in infected tissue are of the most interest. These transcripts likely encode genes responding to the pathogen or genuine factors that underlie genetic resistance, which were broadly grouped into the following categories based on their known roles in other plant systems.

\section{Defense response genes}

Among defense response genes, thaumatin-like protein [17], polygalacturonase-inhibiting protein (PGIP) [32,33], harpin-induced protein-related $[34,35]$, glutaredoxin $[36,37]$ and beta-glucosidase $[38,39]$ have been widely studied in plant pathogen resistance. Thaumatin-like protein, like many other disease resistant proteins [40], is also induced by abiotic stresses, which may indicate existence of a crosstalk between pathogen and abiotic stresses. In this category, tobacco mosaic virus (TMV) response -related protein (+32 fold in INF vs CON) is associated with TMV attack and may also play an important role in DM resistance of grape. 


\section{Transport}

Three transcripts were associated with transport function. Multidrug resistance pump proteins $(+121$ fold in INF vs $\mathrm{CON}$ ) and multidrug resistance $\mathrm{ABC}$ transporter $(+25$ fold in INF vs CON) are well known transporters in clinical study for bacteria infection of human [41]. Such transporters also have been isolated from plants, such as Coptis japonica [42]. They transport several compounds associated with multidrug (antibiotic) resistance which can inhibit pathogen infection in animal model $[41,43]$. Another gene identified to be transport related is mitochondrial dicarboxylate carrier protein $(+38$ fold in INF vs $\mathrm{CON}$ ) which might be involved in the excretion of organic acids and rhizotoxic aluminum tolerance [44].

\section{Signal transduction}

There were fourteen transcripts in our results associated with signal transduction. Two came from genes (GSVIVT00030628001, GSVIVT00030574001) encoding leucine-rich repeat receptor-like protein kinases which were more prevalent (145 and 20 fold) in the INF library than in control. Molecules that indicate the presence of pathogen (elicitors) activate host receptors and that rapidly generate an internal signal that triggers early defense responses [45]. Various signals presented in our results, including phytohormones like ABA and ethylene, as well as intracellular messengers like calcium, phosphoinositide and kinases, have been proposed to regulate plant responses in adverse environmental conditions and thus contribute to the coordination of plant stress physiology [46]. Transcripts representing three kinase-encoding genes (GSVIVT00030628001, GSVIVT00006178001, GSVIVT00019504001) were present 52-145 fold higher in INF than CON, and have been widely documented as signaling factors in many stresses [47-50] and senescence [51]. Four transcripts (GSVIVT00002706001, GSVIVT00020989001, GSVIVT00036549001, GSVIVT00002973001) were found to be more abundant (27 to 39 fold) in INF than $\mathrm{CON}$, and were associated with calcium signaling pathway. All of these are also induced by senescence [52] and many stresses $[53,54]$. Nodulin-like protein $(+23$ fold in INF vs CON) induced in fungal pathogen treatment [55] and drought/heat combination stress [40] has been shown to be involved in salicylic acid (SA) signaling pathway [56]. A RING-H2 gene (+22 fold in INF vs $\mathrm{CON})$ has demonstrated regulatory function in ABA signaling [57], drought tolerance [57], regulation of growth and defense responses against abiotic/biotic stresses [58]. Ethylene-regulated transcript 2 (ERT2) $(+34$ fold in INF vs CON) is involved in ethylene response 'circuit' including ethylene synthesis, perception, signal transduction and regulation of gene expression [59]. The PAR-1a (photoassimilate-responsive) protein $(+22$ fold in INF vs
$\mathrm{CON}$ ) is a serine/threonine kinase with diverse phosphorylation targets and has been reported to be induced by infection with potato virus $\mathrm{Y}[60,61]$.

\section{Transcription}

Eleven transcripts associated with transcription were 21 to 60 fold more abundant in INF than CON libraries. Transcripts annotated as zinc-finger protein 1, DREB protein, AP2 domain class transcription factor, basic helix-loop-helix protein, CBF4(C-repeat binding factor 4), jasmonate ZIM domain 1, GRAS family transcription factor, and WRKY transcription factor 21 were all present at higher steady state levels in infected tissue. They have been documented to play important roles in responding to phytohormone stasis, pathogen attack and environmental stresses [62-69].

\section{Metabolism \\ Synthesis of the hormones}

S-adenosyl-L-methionine (GSVIVT00024884001) and 9cis-epoxycarotenoid dioxygenase 1(NCED1) (GSVIVT00000988001) are transcripts related to synthesis of plant hormones, and were found more frequently (97 and 62 fold, respectively) in the INF library. S-adenosyl-L-methionine is the precursor of ethylene [70] which participates in regulation of growth, development, and responses to stress and pathogen attack in plants [71]. NCED is an important enzyme in synthesizing the phytohormone ABA which plays a central role in responses to pathogen attack [72].

\section{Protein metabolism}

Twelve transcripts related to protein metabolism were more abundant in the INF library, 21 fold to 72 fold. Among them, ubiquitin-protein ligase (GSVIVT00028930001, GSVIVT00035825001), spotted leaf protein (GSVIVT00017518001, GSVIVT00028839001) and f-box family protein (GSVIVT00022245001, GSVIVT00009741001) were identified, and represent proteins involved in ubiquitination and subsequent degradation of target proteins. Aspartic proteinase nepenthesin-1 precursor (GSVIVT00032938001) is expressed at higher level in "Nipponbare" in response to phosphorus deficiency [73] and isolated from salt-stress wild rice "Porteresia coarctata" [74]. Protein phosphatase 2c (GSVIVT00024072001, GSVIVT00024235001, GSVIVT00001432001) regulates numerous ABA responses [75,76]. Nucleic acid binding proteins (GSVIVT00024387001) control genes expression in response to oxidative stress [77], ABA treatment [78] and abiotic stresses [79]. Exocyst subunit EXO70 family protein H4 (GSVIVT00023109001) has been shown to be involved in the exocytic pathway, which sorts newly synthesized proteins from the endoplasmic reticulum to their final destination at the lysosome, vacuole or plasma membrane [80]. 


\section{Secondary metabolism}

This subcategory contained 4 genes, including a higher level of tropinone reductase (GSVIVT00018424001, +48 fold in INF vs CON) transcript in infected leaves, consistent with previous reports showing it to be more abundant after pathogen infection [81]. Isoflavone reductase-like protein 3 (GSVIVT00019233001, +31 fold in INF vs CON) also has a potential pathogen resistance role because it is involved in biosynthesis of isoflavonoid phytoalexins [82], an important product in resistance to pathogen infection [83,84]. UDPglucose glucosyltransferase (GSVIVT00002450001, + 24 fold in INF vs CON) and galactinol synthase (GSVIVT00019669001, + 24 fold in INF vs CON) are reported to be induced by abiotic stresses $[85,86]$.

\section{Cell wall organization}

Three genes were classified into this subcategory. Cellulose synthase-like D1 (GSVIVT00014029001, + 31 fold in INF vs CON) and beta-expansin 1a precursor (GSVIVT00036225001, + 27 fold in INF vs CON) contribute to cell wall synthesis and modification $[87,88]$. The wound-induced protein (WIN2) (GSVIVT00007452001, + 26 fold in INF vs CON) with anti-fungal activity [89] possesses a domain that binds PAMP (pathogen-associated molecular patterns) elicitors (e.g., chitin) [90] and is induced in response to pathogen. In addition, other highly expressed metabolic genes in the INF samples were glucose-1-phosphate adenylyltransferase (GSVIVT00036349001, + 24 fold in INF vs CON), cytochrome P450 (GSVIVT00014730001, + 70 fold in INF vs CON) and serine acetyltransferase (GSVIVT0000$7984001,+30$ fold in INF vs CON). These transcripts are related to carbohydrate metabolism, photosynthesis and cysteine synthesis. Cysteine synthesis has reported to respond to oxidative stress by calcium signaling [91].

Even though most of these genes have been reported to be biotic or abiotic stresses related, seven high expressed genes in the infected leaves have not been previously reported being associated with stress. They were noted as protein-binding protein (GSVIVT00024408001, + 87 fold in INF vs CON), ATPP2-A2 (Arabidopsis thaliana phloem protein 2-A2) (GSVIVT00023$009001,+56$ fold in INF vs CON), putative integral membrane protein (GSVIVT00014704001, + 51 fold in INF vs CON), putative phosphate-induced protein (GSVIVT00015203001, + 229; GSVIVT00015200001, +37 fold in INF vs CON), ATP-dependent DNA helicase (GSVIVT00016166001, +36 fold in INF vs CON), CW14 (GSVIVT00020834001, +23 fold in INF vs CON), and a hypothetical protein (GSVIVT00017533001, +20 fold in INF vs CON).

\section{Transcripts less abundant in infected leaves}

The most striking functions for transcripts less abundant in infected tissue were those associated with metabolism and defense response to pathogen attack. Fifteen DEGs were detected to be less prevalent in the INF libraries more than 20 fold compared to CON, most of which, such as (-)-germacrene D synthase [92], non-specific lipid transfer protein [93], major histocompatibility complex [94], thioredoxin [95], beta-cyano-alanine synthase [96], expansin [97] and UDPglucosyltransferase [98] are reported to be positively associated with plant defense responses to pathogen attack. However, our data indicated that the expression level of these transcripts was lower in infected tissues.

Another two transcripts that were less prevalent in infected tissue (GSVIVT00014727001, -35 fold in INF vs CON; GSVIVT00014725001, -41 in INF vs CON) belong to cytochrome P450 family with oxidative function. Interestingly, a novel gene encoding male sterility-related protein was also identified in this group, and its function associated with DM response has not been clarified.

\section{Conclusions}

Solexa-based sequencing can be used for analyzing variation in gene expression between two samples. The gene expression level in "Zuoshan-1" leaves infected with PV changed significantly in comparison with control leaves. Analysis of differentially-expressed genes involved in the pathogen infection allows delineation of candidate genes potentially relevant to DM resistance in grapevines.

\section{Methods}

\section{Plants material and pathogen infection}

One-year-old, certified virus-free seedlings of "Zuoshan-1" were grown and maintained in the greenhouse under a 16$\mathrm{h}$ light/8-h dark photoperiod at $25^{\circ} \mathrm{C}, 85 \%$ relative humidity. Control plants were maintained under the same conditions. $P$. viticola was collected from sporulated field leaves and used for the artificial inoculations of surface-sterilized leaves. Infections were conducted by dipping the fourth grapevine leaves in a suspension of 10,000 sporangia per $\mathrm{ml}$ pure water. The leaves were covered with plastic bags for one night to ensure high humidity. The fourth unfolded leaf from the shoot apex was harvested from each of three vines, and the three leaves were combined to represent one replicate. Three independent replicates were collected for each sample. Infected leaves were collected every $24 \mathrm{~h}$ for 9 days. Control samples were harvested from water-treated leaves incubated under the same conditions.

\section{Preparation of Digital Expression Libraries}

Samples from infected leaves from $4 \mathrm{~d}$ to $8 \mathrm{~d}$ were pooled for RNA isolation and library construction. Comparable control leaves were treated identically and in parallel. Total RNA was isolated from the leaf mixture using a modification of the CTAB method as presented by Murray and Thompson [99]. Sequence tag 
preparation was done with the Digital Gene Expression Tag Profiling Kit (Illumina Inc; San Diego, CA, USA) according to the manufacturer's protocol (version 2.1B). Six micrograms of total RNA was extracted and mRNA was purified using biotin-Oligo (dT) magnetic bead adsorption. First- and second-strand cDNA synthesis was performed after the RNA was bound to the beads. While on the beads, double strand cDNA was digested with NlaIII endonuclease to produce a bead-bound cDNA fragment containing sequence from the 3'-most CATG to the poly (A)-tail. These 3' cDNA fragments were purified using magnetic bead precipitation and the Illumina adapter 1 (GEX adapter 1) was added to new 5' end. The junction of Illumina adapter 1 and CATG site was recognized by MmeI, which is a Type I endonuclease (with separated recognition sites and digestion sites). The enzyme cuts 17 bp downstream of the CATG site, producing $17 \mathrm{bp}$ cDNA sequence tags with adapter 1. After removing 3' fragments with magnetic bead precipitation, the Illumina adapter 2 (GEX adapter 2) was ligated to 3' end of the cDNA tag. These cDNA fragments represented the tag library.

\section{Solexa sequencing}

Sequencing was performed by "HuaDa Gene" [100] with the method of sequencing by synthesis. A PCR amplification with 15 cycles using Phusion polymerase (Finnzymes, Espoo, Finland) was performed with primers complementary to the adapter sequences to enrich the samples for the desired fragments. The resulting 85 base strips were purified by $6 \%$ TBE PAGE Gel electrophoresis. These strips were then digested, and the singlechain molecules were fixed onto the Solexa Sequencing Chip (flow cell). Each molecule grew into a single-molecule cluster sequencing template through in situ amplification. Four color-labeled nucleotides were added, and sequencing was performed with the method of sequencing by synthesis. Image analysis and basecalling were performed using the Illumina Pipeline, and cDNA sequence tags were revealed after purity filtering. The tags passing initial quality tests were sorted and counted. Each tunnel generates millions of raw reads with sequencing length of $35 \mathrm{bp}$ (target tags plus 3'adaptor). Each molecule in the library represented a single tag derived from a single transcript.

\section{Sequence annotation}

"Clean Tags" were obtained by filtering off adaptor-only tags and low-quality tags (containing ambiguous bases). Comparison of the sequences by blastn was carried out using the following databases: NCBI [101], Genoscope Grape Genome database [25] and VBI Microbial Database [26]. All clean tags were annotated based on grape reference genes. For conservative and precise annotation, only sequences with perfect homology or $1 \mathrm{nt}$ mismatch were considered further. The number of annotated clean tags for each gene was calculated and then normalized to TPM (number of transcripts per million clean tags) $[30,102]$. Sequences were manually assigned to functional categories based on the analysis of scientific literature.

\section{Identification of differentially expressed genes (DEGs)}

A rigorous algorithm to identify differentially expressed genes between two samples was developed [103]. P value was used to test differential transcript accumulation. In the formula below the total clean tag number of the CON library is noted as $\mathrm{N} 1$, and total clean tag number of INF library as N2; gene A holds $\mathrm{x}$ tags in CON and $\mathrm{y}$ tags in INF library. The probability of gene A expressed equally between two samples can be calculated with:

$$
P(y \mid x)=\left(\frac{N 2}{N 1}\right)^{y} \frac{(x+y) !}{x ! y !\left(1+\frac{N_{2}}{N_{1}}\right)^{(x+y+1)}}
$$

FDR (False Discovery Rate) was applied to determine the threshold of $\mathrm{P}$ Value in multiple tests and analyses [104]. An "FDR $<0.001$ and the absolute value of $\log 2-$ Ratio $\geq 1$ " was used as the threshold to judge the significance of gene expression difference.

\section{Real-time RT-PCR analysis}

Samples were prepared using the same method mentioned above and total RNA was isolated from the leaf mixture. Experiments were carried out on three independent biological replicates each containing three technical replicates. First-strand cDNA was synthesized from 650 ng DNase (Promega, Madison, Wisconsin, USA) -treated total RNA using "ImProm-II TM Reverse Transcriptase" (Promega, Madison, Wisconsin, USA) and diluted 20 fold as template. Specific primer pairs of twelve randomly selected genes were designed (Table 4) using Primer Express 3.0 and tested by Real-time RTPCR. Primers specific for $V$. vinifera actin (Forward: AATGTGCCTGCCATGTATGT; Reverse: TCACACCATCACCAGAATCC) were used for the normalization of reactions. Experiments were carried out using Power SYBR Green PCR Master Mix (Applied Biosystems, Warrington, UK) in a StepOne ${ }^{\text {tu }}$ Real-Time PCR System (Applied Biosystems). The reaction volume was $20 \mu \mathrm{l}$, including $10 \mu \mathrm{l}$ Power SYBR Green PCR master mix, 0.9 $\mu \mathrm{l} 10 \mathrm{mM}$ primer, $2.0 \mu \mathrm{l}$ cDNA sample and $6.20 \mu \mathrm{l}$ $\mathrm{dH} 2 \mathrm{O}$. The following thermal cycling profile was used: $95^{\circ} \mathrm{C} 10 \mathrm{~min} ; 40$ cycles of $95^{\circ} \mathrm{C}$ for $15 \mathrm{~s}, 59^{\circ} \mathrm{C}$ for $1 \mathrm{~min}$; $95^{\circ} \mathrm{C}$ for $15 \mathrm{~s}, 60^{\circ} \mathrm{C}$ for $1 \mathrm{~min}, 95^{\circ} \mathrm{C}$ for $15 \mathrm{~s}$. Data were analyzed using StepOne ${ }^{\mathrm{Tu}}$ Software Version 2.0 (Applied Biosystems). Actin expression was used as an internal 
control to normalize all data. The fold change in mRNA expression was estimated using threshold cycles, by the $\Delta \Delta C \mathrm{~T}$ method [105].

\section{Pathway Enrichment Analysis of DEGs}

Pathway enrichment analysis based on KEGG [106] was used to identify significantly enriched metabolic pathways or signal transduction pathways in differentiallyexpressed genes comparing with the whole genome background. The calculating formula is:

$$
\mathrm{P}=1-\sum_{i=0}^{m-1} \frac{\left(\begin{array}{c}
M \\
i
\end{array}\right)\left(\begin{array}{c}
N-M \\
n-i
\end{array}\right)}{\left(\begin{array}{c}
N \\
n
\end{array}\right)}
$$

where $\mathrm{N}$ is the number of all genes that with KEGG annotation, $n$ is the number of DEGs in $N, M$ is the number of all genes annotated to specific pathways, and $\mathrm{m}$ is number of DEGs in $\mathrm{M}$. $\mathrm{Q}$ value was used for determining the threshold of $P$ Value in multiple test and analysis [107]. Pathways with $Q$ value $<0.05$ are significantly enriched in DEGs.

\section{Additional material}

\section{Additional file 1: Complete list of transcripts attributed to $P$.} viticola.

Additional file 2: Complete list of involved pathways for upregualted DEGs. Pathways with $Q$ value $<0.05$ are significantly enriched for upregulated DEGs.

Additional file 3: Complete list of involved pathways for downregualted DEGs. Pathways with $Q$ value $<0.05$ are significantly enriched for downregulated DEGs.

Additional file 4: List of "Zuoshan-1" transcripts upregulated for at least $\mathbf{2}$ fold in INF library. Two fold and more upregualted genes with pathway annotation in INF library were listed in different categories.

Additional file 5: List of "Zuoshan-1" transcripts downregulated for at least $\mathbf{2}$ fold in INF library. Two fold and more downregualted genes with pathway annotation in INF library were listed in different categories.

\section{Abbreviations \\ AFLP: Amplified Fragment Length Polymorphism; BLAST: Basic Local Alignment Search Tool; cDNA: Complementary DNA; CTAB: Hexadecyltrimethylammonium bromide; DEGs: differentially expressed transcripts; NCBI: National Center for Biotechnology Information.}

\section{Acknowledgements}

This research was supported by the "948" Program, Ministry of Agriculture, China (grant no.2006-G26) and National Grape Industry Technology System (grant no.nycytx-30-zy-05). We thank Jun Wang for generous gift of "Zuoshan-1" propagation material, and "HuaDa Gene" for technical assistance throughout the data analysis manuscript preparation.

\section{Author details}

'College of Food Science and Nutritional Engineering, China Agricultural University, Beijing, 100083, China. ${ }^{2}$ Horticultural Sciences Department and the Graduate Program in Plant Molecular and Cellular Biology, University of
Florida, Gainesville, FL, 32611, USA. ${ }^{3}$ School of Information, University of South Florida Tampa, FL, 33620, USA. ${ }^{4}$ Center for Viticulture and Small Fruit Research, Florida A\&M University, Tallahassee, FL, 32317, USA.

\section{Authors' contributions}

JW and YLZ carried out the plant material preparation, PV infection, RNA extraction, preparation of digital expression libraries, sequence analysis, and contributed to data interpretation and manuscript writing. HQZ participated in PV infection and RNA extraction. $\mathrm{HH}$ contributed to sequence analysis. KMF participated in data interpretation and manuscript modification. $J L$ conceived the study, led the experiment design and coordinated all the research activities, contributed to interpretation of the data, manuscript writing and modification. All authors read and approved the final manuscript.

Received: 12 January 2010 Accepted: 28 October 2010 Published: 28 October 2010

References

1. Pearson RC, Goheen AC: Compendium of Grape Diseases. APS Press; 1988.

2. Spencer DM: The Downy mildews. London: Academic Press; 1981.

3. Kiefer B, Riemann M, Buche C, Kassemeyer HH, Nick P: The host guides morphogenesis and stomatal targeting in the grapevine pathogen Plasmopara viticola. Planta 2002, 215:387-393.

4. Staudt $\mathrm{G}$, Kassemeyer HH: Evaluation of downy mildew resistance in various accessions of wild Vitis species. Vitis 1995, 34:225-228.

5. Brown MV, Morre JN, Fenn P, MCNew RW: Evaluation of grape germplasm for downy mildew resistance. Fruit Varieties Journal 1999, 53:22-29.

6. Olmo HP: Vinifera $x$ rotundifolia hybrids as wines grapes. Am J Enol Vitic 1971, 22:87-91.

7. Dai GH, Andary C, Mondolot-Cosson L, Boubals D: Histochemical studies on the interaction between three species of grapevine, Vitis vinifera, $V$. rupestris and $V$. rotundifolia and the downy mildew fungus, Plasmopara viticola. Physiol Mol Plant Pathol 1995, 46:177-188.

8. Unger S, Buche C, Boso S, Kassemeyer HH: The Course of Colonization of Two Different Vitis Genotypes by Plasmopara viticola Indicates Compatible and Incompatible Host-Pathogen Interactions. Phytopathology 2007, 97:780-786.

9. Diez-Navajas AM, Wiedemann-Merdinoglu S, Greif C, Merdinoglu D: Nonhost versus host resistance to the grapevine downy mildew, Plasmopara viticola, studied at the tissue level. Phytopathology 2008, 98:776-780.

10. Merdinoglu D, Wiedemann-Merdinoglu S, Coste P, Dumas V, Haetty S, Butterlin G, Greif C: Genetic Analysis of Downy Mildew Resistance Derived from Muscadinia rotundifolia. Acta Hort 2003, 603:451-456.

11. Henanff GL, Heitz T, Mestre P, Mutterer J, Walter B, Chong J: Characterization of Vitis vinifera NPR1 homologs involved in the regulation of Pathogenesis-Related gene expression. BMC Plant Biology 2009, 9:54-67.

12. Kortekamp A: Expression analysis of defence-related genes in grapevine leaves after inoculation with a host and a non-host pathogen. Plant Physiol Biochem 2006, 44:58-67.

13. Slaughter AR, Hamiduzzaman MM, Gindro K, Neuhaus JM, Mauch-Mani B: Beta-aminobutyric acid-induced resistance in grapevine against downy mildew: involvement of pterostilbene. Eur J Plant Pathol 2008 122:185-195

14. Kini KR, Vasanthi NS, Shetty HS: Induction of beta-1,3-glucanase in Seedlings of Pearl Millet in Response to Infection by Sclerospora graminicola. European Journal of Plant Pathology 2000, 106:267-274.

15. Richter $\mathrm{H}$, Pezet $\mathrm{R}$, Viret $\mathrm{O}$, Gindro K: Characterization of 3 new partial stilbene synthase genes out of over 20 expressed in Vitis vinifera during the Interaction with Plasmopara viticola. Physiological and Molecular Plant Pathology 2006, 67:248-260.

16. Nagarathna KC, Shetty SA, Shetty HS: Phenylalanine Ammonia Lyase Activity in Pearl Millet Seedlings and its Relation to Downy Mildew Disease Resistance. Journal of Experimental Botany 1993, 44:1291-1296.

17. Punja ZK: Genetic engineering of plants to enhance resistance to fungal pathogens-a review of progress and future prospects. Plant Pathol 2001, 23:216-235.

18. Yamamoto $T$, Iketani $H$, leki $H$, Nishizawa $Y$, Notsuka $K$, Hibi $T$, Hayashi $T$, Matsuta N: Transgenic grapevine plants expressing a rice chitinase with 
enhanced resistance to fungal pathogens. Plant Cell Reports 2000, 19:639-646.

19. Vidal JR, Kikkert JR, Wallace PG, Reisch BI: High-efficiency biolistic cotransformation and regeneration of 'Chardonnay' (Vitis vinifera L.) containing npt-Il and antimicrobial peptide genes. Plant Cell Rep 2003, 22:252-260.

20. Bornhoff BA, Harst M, Zyprian E, Topfer R: Transgenic plants of Vitis vinifera cv. Seyval blanc. Plant Cell Rep 2005, 24:433-438.

21. Fan $\mathrm{CH}$, Pu N, Wang XP, Wang YJ, Fang $L, X u$ WR, Zhang JX: Agrobacterium-mediated genetic transformation of grapevine (Vitis vinifera L.) with a novel stilbene synthase gene from Chinese wild Vitis pseudoreticulata. Plant Cell Tiss Organ Cult 2008, 92:197-206.

22. Angarawai II, Kadams AM, Bello D: Gene Effects Controlling Heritability of Downy Mildew Resistance in Nigerian Elite Pearl Millet Lines. World Journal of Agricultural Sciences 2008, 4:545-549.

23. Zyprian E, Welter L, Akkurt M, Ebert S, Salakhutdinov I, Göktürk-Baydar N, Eibach R, Töpfer R: Candidate genes mapping and comparative QTL analysis for powdery and downy mildew resistance in grape. Acta Hort 2009, 827:535-538.

24. Li X, Shen Y, Ge Y, Zang P, Ai J, Jin S: Study for evaluating infecting disease property on plasmopara viticola to the germplasm resources of vitis amurensis rupr. Special Wild Economic Animal and Plant Research 1999, 2:10-13.

25. Genoscope Grape Genome database. [http://www.cns.fr/spip/Nitis-niniferae.html].

26. VBI Microbial Database. [http://phytophthora.vbi.vt.edu/]

27. Keime C, Semon M, Mouchiroud D, Duret L, Gandrillon O: Unexpected observations after mapping LongSAGE tags to the human genome. BMC Bioinformatics 2007, 8:154.

28. Polesani M, Desario F, Ferrarini A, Zamboni A, Pezzotti M, Kortekamp A, Polverari A: CDNA-AFLP analysis of plant and pathogen genes expressed in grapevine infected with Plasmopara viticola. BMC Genomics 2008, 9:142-156.

29. Figueiredo A, Fortes AM, Ferreira S, Sebastiana M, Choi YH, Sousa L, AcioliSantos B, Pessoa F, Verpoorte R, Pais MS: Transcriptional and metabolic profiling of grape (Vitis vinifera L.) leaves unravel possible innate resistance against pathogenic fungi. J Exp Bot 2008, 59:3371-3381.

30. $t$ Hoen PA, Ariyurek $Y$, Thygesen HH, Vreugdenhil E, Vossen RH, de Menezes RX, Boer JM, van Ommen GJ, den Dunnen JT: Deep sequencingbased expression analysis shows major advances in robustness, resolution and inter-lab portability over five microarray platforms. Nucleic Acids Res 2008, 36:e141.

31. Polesani M, Bortesi L, Ferrarini A, Zamboni A, Fasoli M, Zadra C, Lovato A, Pezzotti M, Delledonne M, Polverari A: General and species-specific transcriptional responses to downy mildew infection in a susceptible (Vitis vinifera) and a resistant (V. riparia) grapevine species. BMC Genomics 2010, 11:117.

32. Di Matteo A, Bonivento D, Tsernoglou D, Federici L, Cervone F: Polygalacturonase-inhibiting protein (PGIP) in plant defence: a structural view. Phytochemistry 2006, 67:528-533.

33. Joubert DA, Kars I, Wagemakers L, Bergmann C, Kemp G, Vivier MA, van Kan JA: A polygalacturonase-inhibiting protein from grapevine reduces the symptoms of the endopolygalacturonase BCPG2 from Botrytis cinerea in Nicotiana benthamiana leaves without any evidence for in vitro interaction. Mol Plant Microbe Interact 2007, 20:392-402.

34. Ditt RF, Kerr KF, de Figueiredo P, Delrow J, Comai L, Nester EW: The Arabidopsis thaliana transcriptome in response to Agrobacterium tumefaciens. Mol Plant Microbe Interact 2006, 19:665-681.

35. Jolivet K, Grenier E, Bouchet JP, Esquibet M, Kerlan MC, Caromel B, Mugniery $D$, Lefebvre $V$ : Identification of plant genes regulated in resistant potato Solanum sparsipilum during the early stages of infection by Globodera pallida. Genome 2007, 50:422-427.

36. Ndamukong I, Abdallat AA, Thurow C, Fode B, Zander M, Weigel R, Gatz C: SA-inducible Arabidopsis glutaredoxin interacts with TGA factors and suppresses JA-responsive PDF1.2 transcription. Plant J 2007, 50:128-139.

37. Wang Z, Xing S, Birkenbihl RP, Zachgo S: Conserved functions of Arabidopsis and rice CC-type glutaredoxins in flower development and pathogen response. Mol Plant 2009, 2:323-335.

38. Beffa RS, Neuhaus JM, Meins F Jr: Physiological compensation in antisense transformants: specific induction of an "ersatz" glucan endo-1,3-beta- glucosidase in plants infected with necrotizing viruses. Proc Natl Acad Sci USA 1993, 90:8792-8796.

39. Mattiacci L, Dicke M, Posthumus MA: beta-Glucosidase: an elicitor of herbivore-induced plant odor that attracts host-searching parasitic wasps. Proc Natl Acad Sci USA 1995, 92:2036-2040.

40. Rizhsky L, Liang H, Shuman J, Shulaev V, Davletova S, Mittler R: When defense pathways collide. The response of Arabidopsis to a combination of drought and heat stress. Plant Physiol 2004, 134:1683-1696.

41. Piddock L: Multidrug-resistance efflux pumps - not just for resistance. Nat Rev Microbiol 2006, 4:629-636.

42. Shitan N, Bazin I, Dan K, Obata K, Kigawa K, Ueda K, Sato F, Forestier C, Yazaki K: Involvement of CjMDR1, a plant multidrug-resistance-type ATPbinding cassette protein, in alkaloid transport in Coptis japonica. Proc Natl Acad Sci USA 2003, 100:751-756.

43. Morschhauser J: Regulation of multidrug resistance in pathogenic fungi. Fungal Genet Biol 47:94-106.

44. Deng W, Luo K, Li Z, Yang Y: Molecular cloning and characterization of a mitochondrial dicarboxylate/tricarboxylate transporter gene in Citrus junos response to aluminum stress. Mitochondrial DNA 2008, 19:376-384.

45. Blumwald E, Aharon GS, Lam BCH: Early signal transduction pathways in plant-pathogen interactions. Trends in Plant Science 1998, 3:342-346.

46. Zhu JK: Salt and drought stress signal transduction in plants. Annu Rev Plant Biol 2002, 53:247-273

47. Xiong L, Lee $B$, Ishitani $M$, Lee $H$, Zhang C, Zhu JK: FIERY1 encoding an inositol polyphosphate 1-phosphatase is a negative regulator of abscisic acid and stress signaling in Arabidopsis. Genes Dev 2001, 15:1971-1984.

48. Tang D, Christiansen KM, Innes RW: Regulation of plant disease resistance, stress responses, cell death, and ethylene signaling in Arabidopsis by the EDR1 protein kinase. Plant Physiol 2005, 138:1018-1026.

49. You MK, Oh SI, Ok SH, Cho SK, Shin HY, Jeung JU, Shin JS: Identification of putative MAPK kinases in Oryza minuta and $O$. sativa responsive to biotic stresses. Mol Cells 2007, 23:108-114.

50. Pitzschke A, Schikora A, Hirt H: MAPK cascade signalling networks in plant defence. Curr Opin Plant Biol 2009, 12:421-426.

51. Zhou C, Cai Z, Guo Y, Gan S: An arabidopsis mitogen-activated protein kinase cascade, MKK9-MPK6, plays a role in leaf senescence. Plant Physiol 2009, 150:167-177.

52. Espinoza C, Medina C, Somerville S, Arce-Johnson P: Senescenceassociated genes induced during compatible viral interactions with grapevine and Arabidopsis. J Exp Bot 2007, 58:3197-3212.

53. Knight $\mathrm{H}$ : Calcium signaling during abiotic stress in plants. Int Rev Cytol 2000, 195:269-324.

54. Li A, Wang $\mathrm{X}$, Leseberg $\mathrm{CH}$, Jia J, Mao L: Biotic and abiotic stress responses through calcium-dependent protein kinase (CDPK) signaling in wheat (Triticum aestivum L.). Plant Signal Behav 2008, 3:654-666.

55. Siciliano V, Genre A, Balestrini R, Dewit PJ, Bonfante P: Pre-Penetration Apparatus Formation During AM Infection is Associated With a Specific Transcriptome Response in Epidermal Cells. Plant Signal Behav 2007, 2:533-545.

56. Peleg-Grossman S, Golani Y, Kaye Y, Melamed-Book N, Levine A: NPR1 protein regulates pathogenic and symbiotic interactions between Rhizobium and legumes and non-legumes. PLoS One 2009, 4:e8399.

57. $\mathrm{Ko} \mathrm{JH}$, Yang SH, Han KH: Upregulation of an Arabidopsis RING-H2 gene, XERICO, confers drought tolerance through increased abscisic acid biosynthesis. Plant J 2006, 47:343-355.

58. Liu H, Zhang H, Yang Y, Li G, Wang X, Basnayake BM, Li D, Song F: Functional analysis reveals pleiotropic effects of rice RING-H2 finger protein gene OSBIRF1 on regulation of growth and defense responses against abiotic and biotic stresses. Plant Mol Biol 2008, 68:17-30.

59. Zhong GY, Burns JK: Profiling ethylene-regulated gene expression in Arabidopsis thaliana by microarray analysis. Plant Mol Biol 2003, 53:117-131.

60. Guo $\mathrm{S}$, Kemphues KJ: par-1, a gene required for establishing polarity in $C$. elegans embryos, encodes a putative Ser/Thr kinase that is asymmetrically distributed. Cell 1995, 81:611-620.

61. Herbers K, Monke G, Badur R, Sonnewald U: A simplified procedure for the subtractive CDNA cloning of photoassimilate-responding genes: isolation of cDNAs encoding a new class of pathogenesis-related proteins. Plant Mol Biol 1995, 29:1027-1038.

62. Takatsuji H: Zinc-finger transcription factors in plants. Cell Mol Life Sci 1998, 54:582-596. 
63. Agarwal PK, Agarwal P, Reddy MK, Sopory SK: Role of DREB transcription factors in abiotic and biotic stress tolerance in plants. Plant Cell Rep 2006, 25:1263-1274

64. Pre M, Atallah M, Champion A, De Vos M, Pieterse CM, Memelink J: The AP2/ERF domain transcription factor ORA59 integrates jasmonic acid and ethylene signals in plant defense. Plant Physiol 2008, 147:1347-1357.

65. Duek PD, Fankhauser C: bHLH class transcription factors take centre stage in phytochrome signalling. Trends Plant Sci 2005, 10:51-54

66. Haake V, Cook D, Riechmann JL, Pineda O, Thomashow MF, Zhang JZ: Transcription factor CBF4 is a regulator of drought adaptation in Arabidopsis. Plant Physiol 2002, 130:639-648.

67. Chung HS, Koo AJ, Gao X, Jayanty S, Thines B, Jones AD, Howe GA: Regulation and function of Arabidopsis JASMONATE ZIM-domain genes in response to wounding and herbivory. Plant Physiol 2008, 146:952-964

68. Smit P, Raedts J, Portyanko V, Debelle F, Gough C, Bisseling T, Geurts R: NSP1 of the GRAS protein family is essential for rhizobial Nod factorinduced transcription. Science 2005, 308:1789-1791.

69. Eulgem T, Rushton PJ, Robatzek S, Somssich IE: The WRKY superfamily of plant transcription factors. Trends Plant Sci 2000, 5:199-206.

70. Roje S: S-Adenosyl-L-methionine: beyond the universal methyl group donor. Phytochemistry 2006, 67:1686-1698.

71. Bleecker $A B$, Kende $H$ : Ethylene: a gaseous signal molecule in plants. Annu Rev Cell Dev Biol 2000, 16:1-18.

72. de Torres-Zabala M, Truman W, Bennett MH, Lafforgue G, Mansfield JW Rodriguez Egea P, Bogre L, Grant M: Pseudomonas syringae pv. tomato hijacks the Arabidopsis abscisic acid signalling pathway to cause disease. EMBO J 2007, 26:1434-1443

73. Pariasca-Tanaka J, Satoh K, Rose T, Mauleon R, Wissuwa M: Stress Response Versus Stress Tolerance: A Transcriptome Analysis of Two Rice Lines Contrasting in Tolerance to Phosphorus Deficiency. Rice 2009, 2:167-185.

74. Sengupta S, Majumder AL: Porteresia coarctata (Roxb.) Tateoka, a wild rice: a potential model for studying salt-stress biology in rice. Plant Cell Environ 2010, 33:526-542

75. Gosti F, Beaudoin N, Serizet C, Webb AA, Vartanian N, Giraudat J: ABI1 protein phosphatase $2 \mathrm{C}$ is a negative regulator of abscisic acid signaling. Plant Cell 1999, 11:1897-1910.

76. Vlad F, Rubio S, Rodrigues A, Sirichandra C, Belin C, Robert N, Leung J, Rodriguez $\mathrm{PL}$, Lauriere $\mathrm{C}$, Merlot $\mathrm{S}$ : Protein phosphatases $2 \mathrm{C}$ regulate the activation of the Snf1-related kinase OST1 by abscisic acid in Arabidopsis. Plant Cell 2009, 21:3170-3184.

77. Rodriguez-Gabriel MA, Burns G, McDonald WH, Martin V, Yates JR, Bahler J, Russell P: RNA-binding protein Csx1 mediates global control of gene expression in response to oxidative stress. EMBO J 2003, 22:6256-6266.

78. Razem FA, El-Kereamy A, Abrams SR, Hill RD: The RNA-binding protein FCA is an abscisic acid receptor. Nature 2006, 439:290-294.

79. Kim JS, Jung HJ, Lee HJ, Kim KA, Goh CH, Woo Y, Oh SH, Han YS, Kang H: Glycine-rich RNA-binding protein 7 affects abiotic stress responses by regulating stomata opening and closing in Arabidopsis thaliana. Plant $J$ 2008, 55:455-466.

80. Dong G, Hutagalung AH, Fu C, Novick P, Reinisch KM: The structures of exocyst subunit Exo70p and the Exo84p C-terminal domains reveal a common motif. Nat Struct Mol Biol 2005, 12:1094-1100.

81. Espinoza C, Vega A, Medina C, Schlauch K, Cramer G, Arce-Johnson P: Gene expression associated with compatible viral diseases in grapevine cultivars. Funct Integr Genomics 2007, 7:95-110.

82. Kim ST, Cho KS, Kim SG, Kang SY, Kang KY: A rice isoflavone reductase-like gene, OsIRL, is induced by rice blast fungal elicitor. Mol Cells 2003, 16:224-231.

83. Prasanna TB, Vairamani M, Kasbekar DP: Effects of pisatin on Dictyostelium discoideum: its relationship to inducible resistance to nystatin and extension to other isoflavonoid phytoalexins. Arch Microbiol 1998, 170:309-312.

84. He XZ, Dixon RA: Genetic manipulation of isoflavone 7-Omethyltransferase enhances biosynthesis of 4'-O-methylated isoflavonoid phytoalexins and disease resistance in alfalfa. Plant Cell 2000, 12:1689-1702.

85. Gachon CM, Langlois-Meurinne M, Saindrenan P: Plant secondary metabolism glycosyltransferases: the emerging functional analysis. Trends Plant Sci 2005, 10:542-549.
86. Nishizawa-Yokoi A, Yabuta Y, Shigeoka S: The contribution of carbohydrates including raffinose family oligosaccharides and sugar alcohols to protection of plant cells from oxidative damage. Plant Signal Behav 2008, 3:1016-1018.

87. Hazen SP, Scott-Craig JS, Walton JD: Cellulose synthase-like genes of rice. Plant Physiol 2002, 128:336-340

88. Zheng J, Fu J, Gou M, Huai J, Liu Y, Jian M, Huang Q, Guo X, Dong Z, Wang $H$, et al: Genome-wide transcriptome analysis of two maize inbred lines under drought stress. Plant Mol Biol 72:407-421.

89. Stanford A, Bevan M, Northcote D: Differential expression within a family of novel wound-induced genes in potato. Mol Gen Genet 1989, 215:200-208

90. Ponstein AS, Bres-Vloemans SA, Sela-Buurlage MB, van den Elzen PJ, Melchers LS, Cornelissen BJ: A novel pathogen- and wound-inducible tobacco (Nicotiana tabacum) protein with antifungal activity. Plant Physiol 1994, 104:109-118.

91. Liu F, Yoo BC, Lee JY, Pan W, Harmon AC: Calcium-regulated phosphorylation of soybean serine acetyltransferase in response to oxidative stress. J Biol Chem 2006, 281:27405-27415.

92. Arimura G, Huber DP, Bohlmann J: Forest tent caterpillars (Malacosoma disstria) induce local and systemic diurnal emissions of terpenoid volatiles in hybrid poplar (Populus trichocarpa $\mathrm{x}$ deltoides): CDNA cloning, functional characterization, and patterns of gene expression of (-)-germacrene D synthase, PtdTPS1. Plant J 2004, 37:603-616.

93. Molina A, Segura A, Garcia-Olmedo F: Lipid transfer proteins (nsLTPs) from barley and maize leaves are potent inhibitors of bacterial and fungal plant pathogens. FEBS Lett 1993, 316:119-122.

94. IImonen P, Penn DJ, Damjanovich K, Morrison L, Ghotbi L, Potts WK: Major histocompatibility complex heterozygosity reduces fitness in experimentally infected mice. Genetics 2007, 176:2501-2508.

95. Makino Y, Okamoto K, Yoshikawa N, Aoshima M, Hirota K, Yodoi J, Umesono K, Makino I, Tanaka H: Thioredoxin: a redox-regulating cellular cofactor for glucocorticoid hormone action. Cross talk between endocrine control of stress response and cellular antioxidant defense system. J Clin Invest 1996, 98:2469-2477.

96. Takahashi H, Ishihara T, Hase S, Chiba A, Nakaho K, Arie T, Teraoka T, Iwata M, Tugane T, Shibata D, et al: Beta-cyanoalanine synthase as a molecular marker for induced resistance by fungal glycoprotein elicitor and commercial plant activators. Phytopathology 2006, 96:908-916.

97. Nembaware V, Seoighe C, Sayed M, Gehring C: A plant natriuretic peptidelike gene in the bacterial pathogen Xanthomonas axonopodis may induce hyper-hydration in the plant host: a hypothesis of molecular mimicry. BMC Evol Biol 2004, 4:10.

98. Poppenberger B, Berthiller F, Lucyshyn D, Sieberer T, Schuhmacher R, Krska R, Kuchler K, Glossl J, Luschnig C, Adam G: Detoxification of the Fusarium mycotoxin deoxynivalenol by a UDP-glucosyltransferase from Arabidopsis thaliana. J Biol Chem 2003, 278:47905-47914.

99. Murray MG, Thompson WF: Rapid isolation of high molecular weight plant DNA. Nucleic Acids Res 1980, 8:4321-4325.

100. HuaDa Gene. [http://www.genomics.org.cn/].

101. NCBI. [http://blast.ncbi.nlm.nih.gov/Blast.cgi].

102. Morrissy AS, Morin RD, Delaney A, Zeng T, McDonald H, Jones S, Zhao Y, Hirst M, Marra MA: Next-generation tag sequencing for cancer gene expression profiling. Genome Res 2009, 19:1825-1835.

103. Audic S, Claverie JM: The significance of digital gene expression profiles. Genome Res 1997, 7:986-995.

104. Benjamini Y, Drai D, Elmer G, Kafkafi N, Golani I: Controlling the false discovery rate in behavior genetics research. Behav Brain Res 2001, 125:279-284.

105. Livak KJ, Schmittgen TD: Analysis of relative gene expression data using real-time quantitative PCR and the 2(-Delta Delta C(T)) Method. Methods 2001, 25:402-408.

106. KEGG. [http://www.genome.jp/kegg/]

107. Benjamini $Y$, Hochberg Y: Controlling the false discovery rate: a practical and powerful approach to multiple testing. Journal of the Royal Statistical Society, Series B (Methodological) 1995, 57:289-300.

doi:10.1186/1471-2229-10-234

Cite this article as: Wu et al:: Whole genome wide expression profiles of Vitis amurensis grape responding to downy mildew by using Solexa sequencing technology. BMC Plant Biology 2010 10:234. 